\title{
Thermal Chains and Entrainment in Cumulus Updrafts. Part I: Theoretical Description
}

\author{
Hugh Morrison, ${ }^{\mathrm{a}}$ John M. Peters, ${ }^{\mathrm{b}}$ Adam C. VArble, ${ }^{\mathrm{c}}$ Walter M. Hannah, ${ }^{\mathrm{d}}$ And Scott E. Giangrande ${ }^{\mathrm{e}}$ \\ ${ }^{\mathrm{a}}$ National Center for Atmospheric Research, Boulder, Colorado; ${ }^{\mathrm{b}}$ Department of Meteorology, Naval Postgraduate School, \\ Monterey, California; ${ }^{\mathrm{c}}$ Pacific Northwest National Laboratory, Richland, Washington; ${ }^{\mathrm{d}}$ Lawrence Livermore National \\ Laboratory, Livermore, California; ${ }^{\mathrm{e}}$ Brookhaven National Laboratory, Upton, New York
}

(Manuscript received 10 September 2019, in final form 19 August 2020)

\begin{abstract}
Recent studies have shown that cumulus updrafts often consist of a succession of discrete rising thermals with spherical vortex-like circulations. In this paper, a theory is developed for why this "thermal chain" structure occurs. Theoretical expressions are obtained for a passive tracer, buoyancy, and vertical velocity in axisymmetric moist updrafts. Analysis of these expressions suggests that the thermal chain structure arises from enhanced lateral mixing associated with intrusions of dry environmental air below an updraft's vertical velocity maximum. This dry-air entrainment reduces buoyancy locally. Consequently, the updraft flow above levels of locally reduced buoyancy separates from below, leading to a breakdown of the updraft into successive discrete thermals. The range of conditions in which thermal chains exist is also analyzed from the theoretical expressions. A transition in updraft structure from isolated rising thermal, to thermal chain, to starting plume occurs with increases in updraft width, environmental relative humidity, and/or convective available potential energy. Corresponding expressions for the bulk fractional entrainment rate $\varepsilon$ are also obtained. These expressions indicate rather complicated entrainment behavior of ascending updrafts, with local enhancement of $\varepsilon$ up to a factor of $\sim 2$ associated with the aforementioned environmental-air intrusions, consistent with recent large-eddy simulation (LES) studies. These locally large entrainment rates contribute significantly to overall updraft dilution in thermal chain-like updrafts, while other regions within the updraft can remain relatively undilute. Part II of this study compares results from the theoretical expressions to idealized numerical simulations and LES.
\end{abstract}

KEYWORDS: Convection; Entrainment; Updrafts/downdrafts; Buoyancy; Cumulus clouds

\section{Introduction}

Two basic models have served as a conceptual basis for understanding cumulus convection: steady-state plumes and rising thermals. The canonical plume and thermal models originate from dimensional analysis applied to dry buoyantly driven flows and have been verified in laboratory studies (e.g., Emanuel 1994). In these idealized models there is a constant point source of buoyancy for steady-state plumes, and for thermals a point source of buoyancy that has a finite pulse time.

Dry plumes and thermals differ markedly from one another in their overall structure and entrainment characteristics (notably, about a factor-of-2-greater fractional entrainment for thermals; Morton et al. 1956; Scorer 1957), but neither describes moist cumulus convection particularly well. Latent heating associated with condensation in a conditionally unstable environment is a source of positive buoyancy aloft within moist updrafts. This contrasts with the decrease of buoyancy with height from entrainment for dry plumes and thermals in statically stable or neutral environments. For moist convection, entrainment of dry environmental air leads to evaporation and/or reduced condensation locally, reducing buoyancy relative to that of the surrounding cloudy updraft air. These local buoyancy reductions lead to substantial horizontal heterogeneity within cloudy updrafts. This underlies the "buoyancy sorting" concept (e.g., Raymond and Blyth 1986), whereby engulfment of environmental air and subsequent mixing with cloudy updraft air leads to evaporation; if the

Corresponding author: H. Morrison, hmorrison@ucar.edu mixture becomes negatively buoyant, it is detrained from the updraft and sinks to its level of neutral buoyancy.

Despite the conceptual shortcomings of applying the traditional plume and thermal models to moist convection, no overarching conceptual model for cumulus updrafts as a moist analogy to the dry plume or thermal models has yet been proposed. Convection schemes in atmospheric models are still largely based on the framework of dry steady-state plumes, typically modified post hoc to account for the unique aspects of moist convection (see De Rooy et al. 2013, and references therein). This has included separating entrainment and detrainment associated with organized convective-scale flow (dynamic entrainment and detrainment) from that associated with smaller-scale turbulent mixing (e.g., De Rooy and Siebesma 2010), representing mixing following the buoyancy sorting concept (e.g., Kain and Fritsch 1990; Emanuel 1991; Bretherton et al. 2004), and formulating fractional entrainment rates as a function of various convective parameters. These parameters have included inverse vertical velocity (e.g., Neggers et al. 2002), inverse height (e.g., Jakob and Siebesma 2003), and relative humidity (e.g., Bechtold et al. 2008). An exception to the plume framework for schemes is the stochastic parcel model of Romps (2016).

Although most cumulus schemes are based on the steadystate plume concept, recent large-eddy simulation (LES) studies have indicated that thermal-like structures are common within cumulus updrafts (e.g., Sherwood et al. 2013; Romps and Charn 2015; Hernandez-Deckers and Sherwood 2016; Moser and Lasher-Trapp 2017). For example, LES of tropical deep cumulus clouds showed that they are composed of 


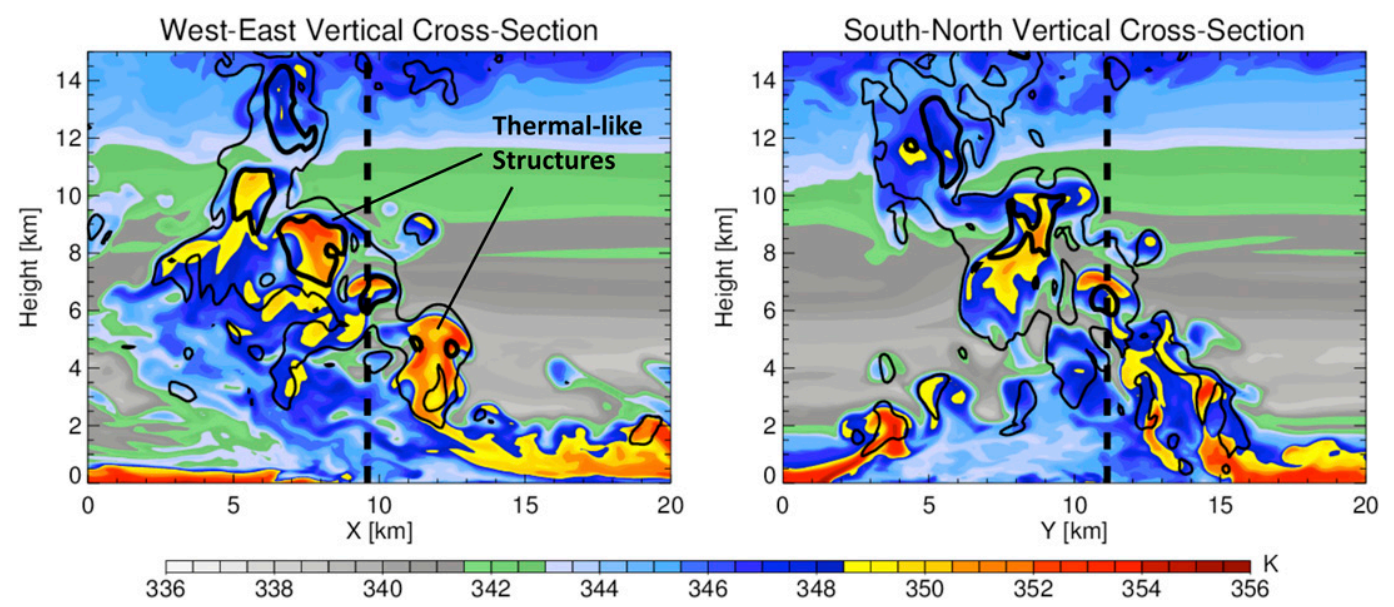

FIG. 1. Perpendicular vertical cross sections through an LES of the 23-24 Jan 2006 deep convection case from the Tropical Warm Pool-International Cloud Experiment with a horizontal grid spacing of $100 \mathrm{~m}$, reproduced from Varble et al. (2014). Equivalent potential temperature $\theta_{e}$ is shaded $(\mathrm{K})$ and vertical velocity is in black contours at intervals of 5 and $15 \mathrm{~m} \mathrm{~s}^{-1}$. The dashed lines show the location of the other cross section. As indicated, distinctive thermal structures rising in succession (the "thermal chain") are seen by the localized areas of large $\theta_{e}$ and vertical velocity between about 2 and $12 \mathrm{~km}$ in height.

clusters of rising buoyant bubbles with spherical vortex-like toroidal circulations (e.g., Sherwood et al. 2013; Romps and Charn 2015; Hernandez-Deckers and Sherwood 2016). Similar organized thermal-like structures have occurred in LES of midlatitude squall lines (e.g., Bryan et al. 2003, 2007; Bryan and Morrison 2012; Peters 2016; Lebo and Morrison 2015). LES of shallow convection have also indicated the prevalence of thermal-like structures (e.g., Heus et al. 2009). Thermals in continental convection have been observed with aircraft-based Doppler radar (Damiani et al. 2006; Damiani and Vali 2007; Damiani et al. 2008) and photogrammetric analyses (Romps and Oktem 2015).

A characteristic feature of the thermal-like cumulus updrafts from LES and observations, particularly for deep convection, is their tendency to occur as successive rising thermals each with their own distinct toroidal circulations (e.g., Raymond and Blyth 1989; Blyth and Latham 1993; Damiani et al. 2006; Kirshbaum 2011; Heus et al. 2009; Moser and Lasher-Trapp 2017; Peters et al. 2019). The succession of rising thermals has implications for the properties of air entrained into later thermals, and may alter precipitation efficiency (Moser and Lasher-Trapp 2017) and contribute to rapid cloud glaciation (Blyth and Latham 1993). We will refer to this structure of cumulus updrafts as a "thermal chain"; an example from LES is shown in Fig. 1. Thermal chains can form a coherent cumulus cloud whose lifetime is often much longer than the individual thermals it comprises, typically of order $1 \mathrm{~h}$ versus 5-10 min. This organized structure of moist thermals gives cumulus convection its characteristic bubbling nature, well encapsulated by Koenig (1963) when describing cumulus congestus observed as part of Project Whitetop in Missouri: "Commonly, these clouds were found to have lifespans on the order of one hour and a pulsating growth habit similar to that described in Scorer and Ludlam's (1953) bubble theory of convection. Each bubble or turret composing the uppermost portion of the cloud was visible generally for 5 to $10 \mathrm{~min}$ : initially, as an active, hard appearing, ascending cloud mass; later, as a dissipating fibrous cloud mass whose place at the summit was soon to be lost to a younger, active bubble."

Although there is ample evidence from LES and observations that cumulus convection is often composed of rising moist thermals in succession, an explanation for why this structure often occurs, rather than plumes or isolated thermals, and under what conditions, is lacking. Because conceptual understanding of moist convection provides the general framework upon which convection schemes are built, improvement in this understanding is directly relevant to improving convection schemes. Better conceptual understanding is also useful for interpreting results from convection-permitting models with horizontal grid spacing $\sim 1-5 \mathrm{~km}$, in which convective updrafts are underresolved. Specifically, these models struggle to reproduce the thermal-like updraft structures found in LES (Varble et al. 2014; Lebo and Morrison 2015). These ideas provide the basic motivation for the work presented herein.

In this paper, we use theory to gain insight into the structure and behavior of thermal chains. Simplified expressions are obtained for the evolution of a passive scalar, buoyancy, and vertical velocity within cumulus updrafts from the governing vertical momentum, mass continuity, and cloud thermodynamic equations. This theoretical analysis builds upon the results of Morrison (2017, hereafter M2017), who proposed that updrafts transition from thermal to plume structure depending on their aspect ratio and the environmental relative humidity. In the current paper, we explain why a thermal chain structure of cumulus updrafts is favored under a certain range of conditions, with a transition from thermal chain to plume-like structure for relatively wide updrafts in a moist environment or isolated thermal-like structure for relatively narrow updrafts in a dry environment. Mechanisms driving this behavior are analyzed, focusing especially on the role of entrainment. 


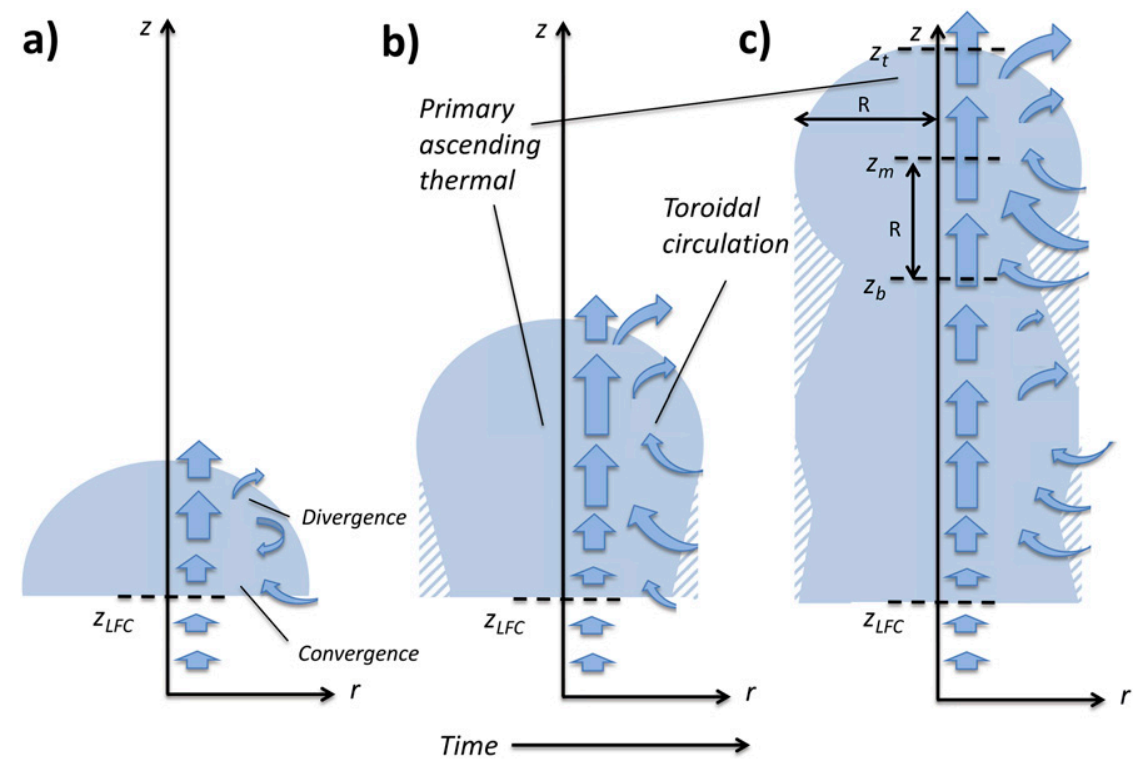

FIG. 2. The conceptual model of moist cumulus updrafts underlying the theoretical derivation. (a)-(c) Different stages of updraft growth above the level of free convection (LFC). The diagrams in (a) and (b) show the ascent of the primary ascending thermal above the LFC and its development of a toroidal circulation. The diagram in (c) shows the development of a second thermal below the primary ascending thermal, and also illustrates the 1:1 aspect ratio assumed for the thermals' toroidal circulations. Solid blue shading indicates the cloudy updraft region, i.e., the region containing cloud water and vertical velocities above $\sim 1 \mathrm{~m} \mathrm{~s}^{-1}$. $R$ indicates the thermal radius, and $z_{t}, z_{m}, z_{b}$, and $z_{\mathrm{LFC}}$ are the heights of the primary thermal top, its center, its bottom, and the LFC, respectively.

We highlight the critical role of feedbacks between the updraft flow structure, localized regions of enhanced dry-air entrainment, and reduction of buoyancy in producing the thermal chain structure. Overall, a simple quantitative model for the structure and behavior of moist convection is developed from this work, going beyond the traditional thermal and plume models and consistent with the idea that thermal chains are a fundamental structural mode of moist convection. A hypothesis regarding updraft structure based on the theoretical model is tested in the companion paper (Peters et al. 2020, hereafter Part II), which compares the theoretical expressions with results from idealized numerical simulations and LES. Though simple, these expressions well capture the behavior of the simulations in Part II.

The rest of the paper is organized as follows. Derivation of the theoretical expressions is given in section 2. In section 3, results from these expressions are described. Implications for bulk fractional entrainment rates are discussed in section 4 . Finally, a summary and conclusions are given in section 5 .

\section{Theoretical expressions}

\section{a. Description of the conceptual model}

To provide context for the expressions obtained below, we first describe the conceptual model underpinning the theory, which is illustrated in Fig. 2. This conceptual model is based in part on the numerical simulations in Part II. An example of the convective structure in these simulations is shown in Fig. 3. Results in this figure were generated from the Cloud Model version 1 (Bryan and Fritsch 2002) in axisymmetric mode, using $100 \mathrm{~m}$ horizontal and vertical grid spacings. Convection was initialized using a warm bubble and a version of the sounding from Weisman and Klemp (1982) modified to have a constant relative humidity of $42.5 \%$ above the level of free convection (LFC). See Part II for simulation details. A thermal chain-like updraft is seen in Fig. 3, with two distinct rising thermals located at heights of about 4 and $7 \mathrm{~km}$ at $18 \mathrm{~min}$. Each thermal contains a toroidal circulation with a radius similar to the updraft as a whole, and local vertical velocity maxima occur near the thermal centers. As shown in Part II, this general structure is consistent with turbulent, three-dimensional LES of convective clouds, although turbulence in these simulations makes the flow structure much more complicated.

Generally speaking, if moist ascending air reaches its LFC, it will be positively buoyant relative to the surrounding environment and accelerate, creating a buoyant cumulus updraft (Fig. 2a). The presence of relatively weak vertical motion in the ambient environment surrounding the updraft implies a pattern of horizontal divergence above the vertical velocity maximum within the updraft, and horizontal convergence below. This flow structure leads to the development of a toroidal circulation within the updraft (Fig. 2b). We refer to this initial toroidal circulation that develops as the "primary ascending thermal." Because cumulus updrafts are turbulent, they 


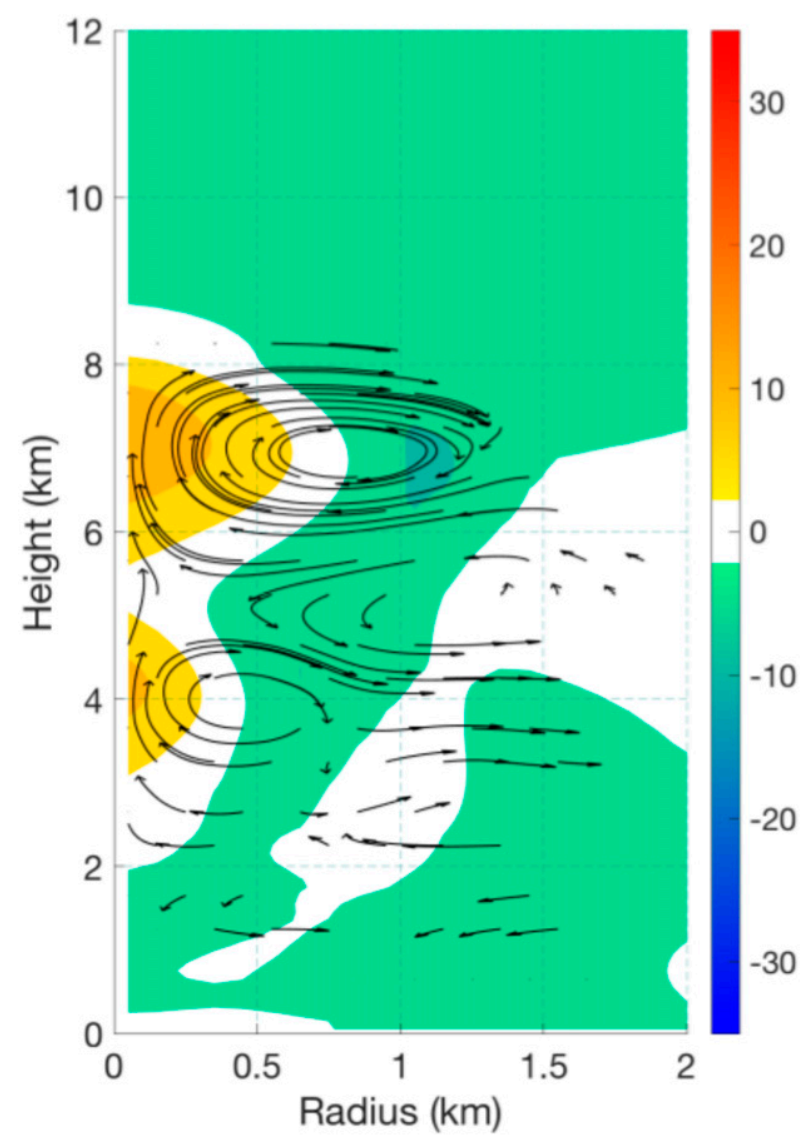

FIG. 3. Example vertical cross section of vertical velocity (color contours $\mathrm{m} \mathrm{s}^{-1}$ ) and flow vectors (black arrows) from one of the axisymmetric model simulations in Part II. "Radius" on the $x$ axis refers to horizontal distance to the central domain axis in the axisymmetric simulation. The initial bubble radius to generate convection is $1400 \mathrm{~m}$, the tropospheric relative humidity is $42.5 \%$, and results are shown at $18 \mathrm{~min}$ into the simulation.

contain thermals and vortices having a wide variety of sizes, from the scale of the updraft itself (as large as several kilometers) all the way down to the Kolmogorov scale. From LES studies, small eddies are much more numerous (HernandezDeckers and Sherwood 2016) while larger thermals carry a significant share of the total upward mass flux (HernandezDeckers and Sherwood 2016; Peters et al. 2019). Here we assume a scale separation between the largest thermals, having a horizontal scale similar to the cloud updraft as a whole, and smaller-scale turbulent eddies. The flow structure of updraft-scale thermals will be considered explicitly in our theoretical model, whereas the effects of smaller-scale eddies on updraft-scale flow will be parameterized using an eddy diffusion approach.

From LES (Romps and Charn 2015; Hernandez-Deckers and Sherwood 2016; Peters et al. 2019), the spherical vortexlike flow structure of moist thermals consists of upward motion along the central vertical axis and thermal-relative descent along the periphery. The maximum vertical velocity $w$ is located near the thermal center, at height $z_{m}$. There is horizontal divergence above $z_{m}$ up to the thermal top, and horizontal convergence below $z_{m}$ down to the thermal bottom. For the axisymmetric updrafts considered here, horizontal wind $u$ must vanish along the vertical axis of symmetry (i.e., at $r=0$ in axisymmetric coordinates, where $r$ is the horizontal distance from the central axis); thus, we neglect the effects of environmental wind shear. Because $u=0$ at $r=0$, the organized inflow of air associated with horizontal convergence near the thermal bottom sharpens horizontal gradients across the updraft, all else equal. The effects of this dynamic entrainment lead to enhanced dilution of updraft properties locally near the thermal bottom, which is consistent with the simulations in Part II and is discussed further in section $2 \mathrm{~b}$. In the divergent flow in the upper part of the thermal, we assume detrained air has properties that are closer to the environment than the updraft core, and thus in effect we neglect the effects of this detrainment in the mathematical framework presented below.

Another key aspect of the thermal circulations within moist updrafts is their tendency to retain an approximately constant radius and spherical shape as they rise, so long as they remain positively buoyant. This behavior is evident from LES (Hernandez-Deckers and Sherwood 2016, 2018), and is incorporated into the conceptual model as illustrated in Fig. 2c. Note, however, that once significant negative buoyancy is generated within thermals with $R>1 \mathrm{~km}$ as they rise through the upper troposphere, their radii increase rapidly (see Part II). For simplicity this behavior is neglected, and we assume constant $R$ as thermals rise.

Finally, we assume that there is sustained ascent to the LFC beneath the primary ascending thermal. This allows for the potential development of additional thermals under the primary thermal, or a starting plume-like updraft ${ }^{1}$ with continuous upward acceleration of air from the LFC to the primary ascending thermal (Fig. 2c). Ascent to the LFC can be maintained by upward motion associated with features such as mesoscale boundaries or topography. However, the upwarddirected vertical buoyant pressure gradient force below moist thermals can itself induce sustained low-level convergence and ascent through perturbation pressure forcing in the absence of any external forcing mechanisms. This mechanism is discussed in Brown and Nowotarski (2019) and Leger et al. (2019).

\section{b. Derivation of the theoretical expressions at the center and top of the primary ascending thermal}

Based on the aforementioned conceptual model, we first develop expressions for a passive scalar $C$ and buoyancy $B$ at the top of the primary thermal, height $z_{t}$, as it ascends along the central axis of the updraft $(r=0)$. We then derive an expression for $w$ at the center of the primary ascending thermal, at height $z_{m}$. In the following, all heights are with respect to the LFC which is defined to have a height of zero in this coordinate system. This yields mathematically identical expressions to

\footnotetext{
${ }^{1}$ The "starting plume," first proposed by Turner (1962), consists of an ascending plume head containing a toroidal circulation - the primary ascending thermal-with flow similar to a steady-state plume behind the primary ascending thermal.
} 
those derived using absolute heights, but simpler to write. We also calculate quantities at $r=0$ because this simplifies the derivation owing to the axisymmetry, specifically, the fact that $u=0$ at $r=0$. Because $\partial w / \partial z<0$ between $z_{m}$ and $z_{t}$, dynamic entrainment is not considered for this divergent flow. The effects of dynamic entrainment are considered at the thermal bottom where there is horizontal convergence, in section $2 \mathrm{~b}$. Overall, this approach is similar to M2017, who derived approximate analytical solutions for $C, B$, and $w$ along $r=0$ at the height of maximum buoyancy of a rising updraft. However, in contrast to M2017 we explicitly consider 1) the role of differences between a thermal's ascent rate and the local vertical velocity within it associated with the thermal's toroidal circulation; and 2) the fact that maximum vertical velocity occurs near the thermal center (Morrison and Peters 2018), well below updraft top and typically below the height of maximum buoyancy. The latter is important because the height of maximum $w$ defines levels of horizontal divergence and convergence.

To obtain a theoretical expression for evolution of $C$ at height $z_{t}$, we start with the Reynolds-averaged scalar conservation equation in axisymmetric cylindrical coordinates, neglecting molecular diffusion:

$$
\frac{d \bar{C}}{d t}=\frac{\partial \bar{C}}{\partial t}+\bar{u} \frac{\partial \bar{C}}{\partial r}+\bar{w} \frac{\partial \bar{C}}{\partial z}=-\underbrace{\frac{1}{r} \frac{\partial\left(r \overline{u^{\prime} C^{\prime}}\right)}{\partial r}}_{\text {Term A }}-\underbrace{\frac{\partial\left(\overline{w^{\prime} C^{\prime}}\right)}{\partial z}}_{\text {Term B }},
$$

where $t$ is time, overbars denote the organized thermal-scale flow, and primes denote smaller-scale turbulent perturbations from this flow (note this equation is general and applies at any location and time). The background environment is assumed to be motionless. Hereafter, overbars are omitted for nonprime quantities. These nonprime quantities correspond to the organized convective-scale flow unless stated otherwise.

Term B represents the vertical turbulent flux divergence, hereafter neglected for simplicity. This assumes the dominant role of lateral mixing, consistent with some recent parceltracking LES studies (Heus et al. 2008; Böing et al. 2014). We note that vertical mixing may be enhanced at $z_{t}$ owing to large vertical derivatives that often occur near the thermal top. However, this mixing is difficult to generalize because it depends on the exact location of $z_{t}$ relative to vertical gradients in the $C$ field; there is no precise definition of thermal top and different studies have applied different methods to define this in analyzing moist thermal simulations (e.g., Sherwood et al. 2013; Romps and Charn 2015). Furthermore, values of $C$ (and $B)$ at $z_{t}$ are quantitatively similar to numerically simulated values as shown in Part II, which suggests that explicitly representing vertical mixing is not critical for evolving these quantities.

Term A represents the horizontal turbulent flux divergence. This term is evaluated at $r=0$ and approximated by its horizontal average from $r=0$ to $r=L$, where $L$ is a turbulent mixing length, giving $(1 / r) \partial\left(r \overline{u^{\prime} C^{\prime}}\right) / \partial r \approx(2 / L)\left(\overline{u^{\prime} C^{\prime}}\right)_{L}$ [Eq. (4) in M2017]. The subscript $L$ indicates the value of $\overline{u^{\prime} C^{\prime}}$ at $r=L$, and $\left(\overline{u^{\prime} C^{\prime}}\right)_{L}$ is approximated using a simple first-order eddy diffusion approach [Eq. (5) in M2017], implying that

$$
\frac{1}{r} \frac{\partial\left(r \overline{u^{\prime} C^{\prime}}\right)}{\partial r} \approx \frac{2}{L}\left(\overline{u^{\prime} C^{\prime}}\right)_{L} \approx-\frac{2 K_{C}}{L} \frac{\partial C}{\partial r}
$$

where quantities on the right-hand side of (2) are evaluated at $r=L$. The turbulent diffusion coefficient $K_{C}$ is a function of the deformation and a turbulent mixing length $L: K_{C}=\left(k^{2} L^{2} / P_{r}\right)|\partial w / \partial r|$ [Eq. (6) in M2017], where $k$ is a mixing coefficient and Pr is the Prandtl number. Following Asai and Kasahara (1967), only the horizontal component of the shear stress is included. Horizontal derivatives of $C$ and $w$ at $z_{t}$ and $r=L$ are approximated by a simple linear difference operator between values at the updraft lateral edge $(r=R)$ and its center $(r=0)$. This gives $\partial w / \partial r \approx-w_{t} / R$ and $-\partial C / \partial r \approx-\left(C_{t}-C_{E}\right) / R$, where $C_{t}$ and $w_{t}$ are the scalar and vertical velocity, respectively, at $z_{t}$ and $r=0, C_{E}$ is the scalar value in the environment at $r=R$, and $w=0$ at $r=R$.

Using the chain rule, we make the substitution $d C_{t} / d t=$ $\left(d C_{t} / d z_{t}\right)\left(d z_{t} / d t\right)$. In this case, $d C_{t} / d z_{t}$ represents the change in $C_{t}$ as a function of the thermal top height as it rises, and $W \equiv d z_{t} / d t$ is the thermal's ascent rate. For a thermal to be coherent, its top must ascend at the rate of the thermal as a whole, meaning that $W=w_{t}$. Thus, we may write $d C_{t} / d t=w_{t} d C_{t} / d z_{t}$. Combining the approximations above with (1), dividing by $w_{t}$, and rearranging terms yields

$$
\frac{d C_{t}}{d z_{t}}=-\frac{2 k^{2} L}{P_{r} R^{2}}\left(C_{t}-C_{E}\right)
$$

Equation (3) is an estimate of the decrease of $C_{t}$ owing to lateral mixing of updraft and environment air as the thermal ascends. Using

$$
\varepsilon=\frac{2 k^{2} L}{P_{r} R^{2}}
$$

we may express (3) as

$$
\frac{d C_{t}}{d z_{t}}=-\varepsilon\left(C_{t}-C_{E}\right)
$$

This has the same form as the expression for scalar transport in the steady-state bulk plume model (e.g., Betts 1975), if steadystate is assumed so that $\partial / \partial t=0$. Expressions derived later for $B$ and $w$ at the center or top of the primary ascending thermal that incorporate $\varepsilon$ are also similar to those solved within plume-based convection parameterizations, except that they include some additional simplifications to obtain analytical solutions while including a more explicit representation of the pressure forcing term. Note that here $\varepsilon$ is an implied fractional entrainment rate; that is, it is the fractional entrainment rate needed to produce evolution of $C_{t}$ consistent with (5). We emphasize that such bulk ${ }^{2}$ estimates of $\varepsilon$ generally differ from direct calculations of $\varepsilon$ that make no assumptions about the properties of entrained air (Romps 2010); see section 4 for

\footnotetext{
${ }^{2}$ In this context "bulk" means that we assume all entrained air at a given height has characteristics of the far-field background environment at that height.
} 
further discussion. Also note that we define $\varepsilon$ consistent with the dilution of $C_{t}$ at $r=0$, rather than a horizontal average across the updraft as is typically done using the bulk plume approach to estimate $\varepsilon$. This is a reasonable assumption; see Part II for details.

Equation (5) can be easily solved by integrating along the path of the rising thermal as its top ascends from the LFC to height $z_{t}$, provided a lower boundary condition of $C_{t}=C_{\mathrm{LFC}}$ at the LFC and assuming $C_{E}$ is constant with height. Using standard notation for integration using dummy variables, we rearrange (5) and integrate as the thermal top rises from $z_{\mathrm{LFC}}=0$ to $z_{t}$ to give

$$
\int_{C_{\mathrm{LFC}}}^{C_{t}} \frac{d C_{t}^{*}}{\left(C_{t}^{*}-C_{E}\right)}=\int_{0}^{z_{t}}-\varepsilon d z_{t}^{*} .
$$

Evaluating the integrals in (6) and rearranging terms yields the exponential solution

$$
C_{t}=\left(C_{\mathrm{LFC}}-C_{E}\right) e^{-\varepsilon z_{t}}+C_{E}
$$

For $C_{E}<C_{\mathrm{LFC}}$, (7) gives an exponential decrease of the scalar at the thermal top as it ascends. However, this approach cannot be used to derive an expression for buoyancy at the thermal top $B_{t}$ because the equation for $B_{t}$ cannot be analytically integrated. Thus, we develop an alternative approach to obtain an approximate solution for $C_{t}$ that is subsequently used to derive an expression for $B_{t}$ later. This is obtained by approximating $C_{t}^{*}$ in the denominator on the left-hand side of (6) with an estimate of its vertical average between $z_{t}^{*}=z_{\mathrm{LFC}}$ and $z_{t}^{*}=z_{t}$ given by $C_{t}^{*} \approx\left(C_{\mathrm{LFC}}+C_{t}\right) / 2$. Using this approximation in (6), evaluating the integrals, and rearranging terms yields

$$
C_{t} \approx \frac{\left(1-\frac{\varepsilon z_{t}}{2}\right) C_{\mathrm{LFC}}+\varepsilon z_{t} C_{E}}{1+\frac{\varepsilon z_{t}}{2}}
$$

This is a close approximation of the exact analytic exponential solution given by (7); errors are generally less than a few percent. As noted in M2017, the accuracy of this approximation is explained by the fact that (8) with $C_{E}=0$ has the same form as a truncated approximation of the power series for $e^{-x}$ valid when $x \ll 2$; that is, $e^{-x} \approx(1-x / 2) /(1+x / 2)$. For the tracer analysis hereafter we will use (8) and assume $C_{E}=0$.

We use a similar approach to derive an expression for buoyancy at the top of the primary ascending thermal, $B_{t}$. Following M2017 [Eqs. (12)-(23)], an expression for condensation rate is derived as a function of $w_{t}$ and the lateral turbulent mixing of temperature $T$ and water vapor mixing ratio $q_{v}$ is treated similarly to the mixing of $C$ [Eq. (15) in M2017]. From the condensation rate, we obtain expressions for the local latent heating and the buoyancy tendency $d B_{t} / d t$ at the thermal top as it ascends [following Eqs. (16) and (17) in M2017]. Applying similar approximations to the expression for $d B_{t} / d t$ as used above to derive (8) gives the following expression for $B_{t}$ (see M2017 for a full derivation):

$$
B_{t}=B_{\mathrm{AD}}-\frac{\varepsilon z_{t}}{2} B_{t}-\varepsilon \Omega
$$

where $B_{\mathrm{AD}}$ is the undilute buoyancy for an air parcel that undergoes pseudoadiabatic moist ascent, and neglecting the contribution from condensate loading. Note this is identical to Eq. (22) in M2017 but written in a more compact form. The parameter $-\left(\varepsilon z_{t} / 2\right) B_{t}$ represents the effects of turbulent mixing of environmental temperature on buoyancy, whereas the effects of dry-air entrainment on buoyancy are represented by the parameter $\Omega \equiv \int_{z_{\mathrm{LFC}}}^{z_{t}}\left(H_{v} g / c_{p}\right)\left\{q_{s, E}\left(1-R_{H}\right) /\left[T_{E}\left(1+H_{v}^{2} q_{s, E} / c_{p} R_{v} T_{E}^{2}\right)\right]\right\} d z_{t}^{*}$, where $H_{v}$ is the latent heat of condensation, $g$ is the acceleration of gravity, $c_{p}$ is the specific heat of air at constant pressure, $R_{v}$ is the gas constant for water vapor, $R_{H}$ is the environmental relative humidity, $T_{E}$ is the environmental temperature, and $q_{s, E}$ is the environmental saturation vapor mixing ratio. This derivation neglects the effects of condensate loading on buoyancy, and any impacts of ice microphysics (e.g., latent heat of fusion) for analytic tractability.

Solving for $B_{t}$ gives

$$
B_{t}=\frac{B_{\mathrm{AD}}-\varepsilon \Omega}{1+\frac{\varepsilon z_{t}}{2}},
$$

which is the buoyancy at the rising thermal top. Note that depending on the environmental $R_{H}, B_{t}$ from (10) can be negative in the case of a dry background environment. For simplicity, we assume an unlimited supply of cloud water is available for evaporation as cloud water mixing ratio is not included within the equation set. In reality, the production of negative buoyancy through evaporation is limited, of course, by the available cloud water. It is apparent from (10) that as $\varepsilon \rightarrow 0, B_{t} \rightarrow B_{\mathrm{AD}}$, meaning that vanishing entrainment leads to undilute adiabatic buoyancy in the thermal core as is physically reasonable. Likewise as $\varepsilon$ becomes large in a moist environment (so that $\Omega=0$ ), the denominator of (10) becomes large resulting in vanishing buoyancy in the thermal, meaning that large entrainment rates cause the thermal's buoyancy to mix out. Finally, as $\varepsilon$ becomes large and $R_{H}$ decreases, $\varepsilon \Omega$ becomes large and may result in $B_{t}<0$. This reflects the progressively deleterious effects of dry-air entrainment on buoyancy. All of these behaviors with respect to $\varepsilon$ and $R_{H}$ are physically reasonable.

We next derive an expression for $w$ at the center of the primary ascending thermal, at height $z_{m}$, which is the approximate location of maximum $w$ within the thermal based on LES (e.g., Sherwood et al. 2013; Romps and Charn 2015). Although the Boussinesq approximation is strictly valid for scales of motion much smaller than the atmospheric scale height $(\sim 8 \mathrm{~km})$, it is assumed here for tractability. Morrison (2016b) compared idealized updrafts in Boussinesq and anelastic frameworks. That study showed a large impact of the Boussinesq approximation on the perturbation pressure field (up to a factor of $\sim 2$ difference in the mean vertical perturbation pressure gradient between the top and bottom of deep updrafts), but little impact on maximum $w$ (less than a few percent). This was explained by the fact that although vertical gradients of perturbation pressure scale approximately with $\rho$, there is a compensation in the vertical momentum equation because the vertical gradients of perturbation pressure in the pressure forcing term are multiplied by $\rho^{-1}$ when solving for $w$ 
[see Morrison (2016b) for a detailed discussion]. We perform Reynolds-averaging of the inviscid vertical momentum equation, neglecting the vertical turbulent flux divergence and approximating the horizontal turbulent flux $\overline{u^{\prime} w^{\prime}}$ using an approach similar to that for $C$. This gives the following vertical momentum equation, written as a Lagrangian derivative for a parcel rising along the vertical axis of symmetry:

$$
\frac{d w}{d t}=w \frac{d w}{d z}=B-\frac{1}{\rho_{0}} \frac{\partial p_{b}}{\partial z}-\frac{1}{\rho_{0}} \frac{\partial p_{d}}{\partial z}-P_{r} \varepsilon w^{2} .
$$

Here $p_{b}$ is the buoyant perturbation pressure that depends on the structure of the buoyancy field, and $p_{d}$ is the dynamic perturbation pressure that depends primarily on the wind field (e.g., Markowski and Richardson 2010). The $-P_{r} \varepsilon w^{2}$ term represents the effects of lateral turbulent momentum mixing. Similar to the derivation of (3), we use the chain rule to give $d w / d t=(d w / d z)(d z / d t)=w d w / d z$ where $z$ is the height of a parcel rising along $r=0$ and $d w / d z$ is the change in $w$ as a function of the parcel height.

Previous studies (e.g., Morrison 2016a,b; Peters 2016; Jeevanjee 2017) have shown that the maximum vertical velocity is well approximated by vertically integrating the vertical momentum equation from updraft bottom to top (defined by its buoyancy field) while neglecting dynamic pressure forcing, particularly for weakly to moderately sheared environments. This is explained by an approximately symmetric dynamic pressure field about a horizontal axis through updraft center with negative vertical gradients (upward forcing) in the lower part and positive gradients (downward forcing) in the upper part of the updraft. Although these studies assumed, either implicitly or explicitly, a steady-state Eulerian framework, this is a reasonable approach from the standpoint of integrating over the path of a rising parcel in a Lagrangian framework since this structure of the dynamic pressure field is general and persists over the lifetime of rising updrafts (Peters 2016). Following this idea, we integrate the Lagrangian vertical momentum equation, (11), neglecting dynamic pressure forcing, along the trajectory of a parcel rising along the thermal's axis of symmetry from the LFC to near $z_{t}$ to estimate $w_{m}$. This trajectory rises both with the thermal (since the thermal as a whole rises over time) and through the thermal (since individual parcels rise through the thermal relative to the thermal's ascent rate), thus encompassing most or all of the upward buoyant forcing experienced by parcels rising from the LFC through the updraft (Morrison and Peters 2018). ${ }^{3}$ Note that parcel trajectories through the thermal must end near, but not precisely at, $z_{t}$ since our framework assumes a parcel at the thermal top rises with the thermal as a whole. For simplicity we extend the upper bound of integration to $z_{t}$ itself.

\footnotetext{
${ }^{3}$ Note that thermals ascending above their levels of neutral buoyancy generate negative buoyancy near thermal top that leads to downward buoyant forcing of rising parcels, but this effect is neglected here. Because of dilution with environmental air, this level of neutral buoyancy is generally lower than that of an adiabatically rising parcel for a given environmental thermodynamic sounding.
}

Although dynamic pressure forcing has a small impact on the magnitude of maximum $w$ except in strongly sheared environments, and we neglect it in estimating $w_{m}$, it does have one critical effect: the upward-directed dynamic pressure forcing lower in thermals and downward-directed forcing in the upper part explains why the maximum vertical velocity occurs near height $z_{m}$ rather than $z_{t}$ even when the effective buoyant forcing is maximized near $z_{t}$ (Morrison and Peters 2018). Because the maximum $w$ occurs near $z_{m}$ and not $z_{t}$, parcels undergo mixing for less time to reach their height of maximum $w$ near $z_{m}$ compared to their full ascent through the thermal to near $z_{t}$. This physical argument suggests that the mixing term in (11) should be integrated from the LFC to $z_{m}$ rather than to $z_{t}$ to estimate $w_{m}$, although whether it is integrated to $z_{m}$ or $z_{t}$ has negligible impact on results. Because of the divergent flow between $z_{m}$ and $z_{t}$, the effects of dynamic entrainment are not considered for $w_{m}$ and the $\varepsilon$ from (4) is used, similar to the derivation of $C$ and $B$ at $z_{t}$. This also implies we can use $B_{t}$ to approximate the buoyancy in (11).

With these assumptions and approximations, integrating (11) from $z_{\mathrm{LFC}}$ to $z_{t}$ (or $z_{m}$ for the mixing term) gives

$$
\frac{w_{m}^{2}}{2}=\int_{z_{\mathrm{LFC}}}^{z_{t}} B_{t} d z^{*}-\int_{z_{\mathrm{LFC}}}^{z_{t}} \frac{1}{\rho_{0}} \frac{\partial p_{b}}{\partial z} d z^{*}-\int_{z_{\mathrm{LFC}}}^{z_{m}} P_{r} \varepsilon w^{2} d z^{*} .
$$

The effective buoyancy [sum of the first and second terms on the right-hand side of (12)] is approximated from Morrison (2016a), where $\int_{z_{\mathrm{LFC}}}^{z_{t}}\left[-\left(1 / \rho_{0}\right)\left(\partial p_{b} / \partial z\right)+B_{t}\right] d z^{*} \approx(1+$ $\left.2 \alpha^{2} R^{2} / z_{t}^{2}\right)^{-1} \int_{z_{\mathrm{LFC}}}^{z_{t}} B_{t} d z^{*}$, with $z_{\mathrm{LFC}}=0$. Here $\alpha$ is the ratio of $w$ at $r=0$ to $w$ horizontally averaged across the updraft. The mixing term [third term on the right-hand side of (12)] is calculated by assuming a linear increase of $w$ as a parcel rises between $z_{\text {LFC }}$, where $w=0$, and $z_{m}$, where $w=w_{m}$. This implies $w \approx w_{\mathrm{LFC}}+\left[\left(w_{m}-w_{\mathrm{LFC}}\right)\left(z^{*}-z_{\mathrm{LFC}}\right) /\left(z_{m}-z_{\mathrm{LFC}}\right)\right]=w_{m} z^{*} / z_{m}$. From this, it follows $\int_{z_{\mathrm{LFC}}}^{z_{m}} w^{2} d z^{*} \approx\left(w_{m}^{2} / z_{m}^{2}\right) \int_{z_{\mathrm{LFC}}}^{z_{m}} z^{* 2} d z^{*}=w_{m}^{2} z_{m} / 3$. With these approximations, (12) is expressed as follows, after rearranging terms:

$$
w_{m}=\sqrt{2\left(1+\frac{2 \alpha^{2} R^{2}}{z_{t}^{2}}\right)^{-1}\left(1+\frac{2}{3} P_{r} \varepsilon z_{m}\right)^{-1} \int_{z_{\mathrm{LFC}}}^{z_{t}} B_{t} d z^{*}} .
$$

In contrast to M2017, who made additional approximations to the buoyancy term in order to obtain fully analytical solutions for $w$ [Eq. (26) therein], we retain a more general form that expresses the buoyancy term as a vertical integral. Here and in Part II this term is found by numerical integration of the analytic expression for $B_{t}$ given by (10) from $z_{\mathrm{LFC}}$ to $z_{t}$ as the thermal rises. Otherwise this expression is similar to M2017.

Equation (13) has physically consistent behavior with regard to the effects of buoyant perturbation pressure forcing and entrainment/mixing. As updrafts become narrow relative to their depth, the perturbation pressure term $\alpha^{2} R^{2} / z_{t}^{2} \rightarrow 0$ and there is little reduction in $w_{m}$ from buoyant pressure forcing consistent with previous studies (e.g., Morrison 2016a; Peters 2016; Jeevanjee 2017). Conversely, when $\alpha^{2} R^{2} / z_{t}^{2} \rightarrow \infty, w_{m} \rightarrow$ 0 as the hydrostatic limit is approached. As $\varepsilon$ is increased, $w_{m}$ becomes smaller both through momentum mixing and the reduction of buoyancy, though as will be shown in section 3 the 
latter generally has a much greater influence on $w$. Similarly, $w_{m}$ is smaller when the environmental $R_{H}$ is reduced, owing to the reduction of buoyancy. When both perturbation pressure effects and mixing are neglected, the latter by setting $\varepsilon=0$, (13) simply reverts to the theoretical maximum $w$ of an adiabatic rising parcel given by $w_{\max }=\sqrt{2 \mathrm{CAPE}}$, where convective available potential energy is CAPE $=\int_{z_{\mathrm{LFC}}}^{z_{t}} B_{\mathrm{AD}} d z^{*}$.

\section{c. The role of dynamic entrainment on mixing}

In our conceptual model, convergence and net lateral inflow occur where $\partial w / \partial z>0$ below the maximum $w$ at height $z_{m}$ within the primary ascending thermal (Fig. 2c). Air that is horizontally converged just below $z_{m}$ is mostly recirculated from within the thermal, and hence is expected to have thermodynamic properties closer to the updraft than the environment. In contrast, horizontal convergence at the thermal bottom (height $z_{b}$ ) is associated with the inflow of mostly environmental rather than thermal air. This dynamic entrainment sharpens horizontal gradients because horizontal advection associated with organized flow cannot directly dilute properties along $r=0 ; u=0$ at $r=0$ as noted in section 2a. Increased horizontal gradients in turn enhance the effects of turbulent lateral mixing along $r=0$ near $z_{b}$.

Below, simple expressions are derived to account for the effects of dynamic entrainment at height $z_{b}$. We start with the conservation equation for a passive scalar $C$ following the primary ascending thermal in axisymmetric coordinates. For now the effects of turbulent mixing are neglected, giving

$$
\frac{D C}{D t}=-u \frac{\partial C}{\partial r}-(w-W) \frac{\partial C}{\partial z},
$$

where all quantities correspond to the organized thermal-scale flow. The left-hand side of (14) is a total derivative following the thermal's ascent rate $W$, and $w-W$ represents vertical motion relative to the thermal's ascent. Here we define $D / D t \equiv$ $\partial / \partial t+W \partial / \partial z$ as a total derivative following the rising thermal as a whole.

Similar to Asai and Kasahara (1967), De Rooy and Siebesma (2010), and M2017, $u \partial C / \partial r$ is approximated by a horizontal upwind finite difference to give

$$
\left(\frac{D C}{D t}\right)_{R / 2} \approx-\frac{2 u_{R / 2}\left(C_{R}-C_{R / 2}\right)}{R}-w_{R / 2}^{\#} \frac{\partial C_{R / 2}}{\partial z},
$$

where $w_{R / 2}^{\#}=w_{R / 2}-W$, subscript " $R$ " indicates quantities at the lateral updraft boundary (i.e., along $r=R$ ) and subscript " $R / 2$ " indicates the quantities at the updraft half width (i.e., along $r=R / 2$ ). A diagram of the conceptual model and discretization used for this derivation is provided in Fig. 4.

We approximate $w_{R / 2}^{\#} \approx \widehat{w^{\#}}$, where $\widehat{w^{\#}}$ is the thermal-relative vertical velocity horizontally averaged across the thermal. By definition $\widehat{w^{\#}}=0$ in the reference frame following the thermal when there is no change in thermal size or shape. Thus, the second term on the right-hand side of (15) vanishes. We again note that $u=0$ at $r=0$ by symmetry, and assuming a linear horizontal $u$ profile this gives $u_{R / 2} \approx u_{R} / 2$. From this, we express (15) as

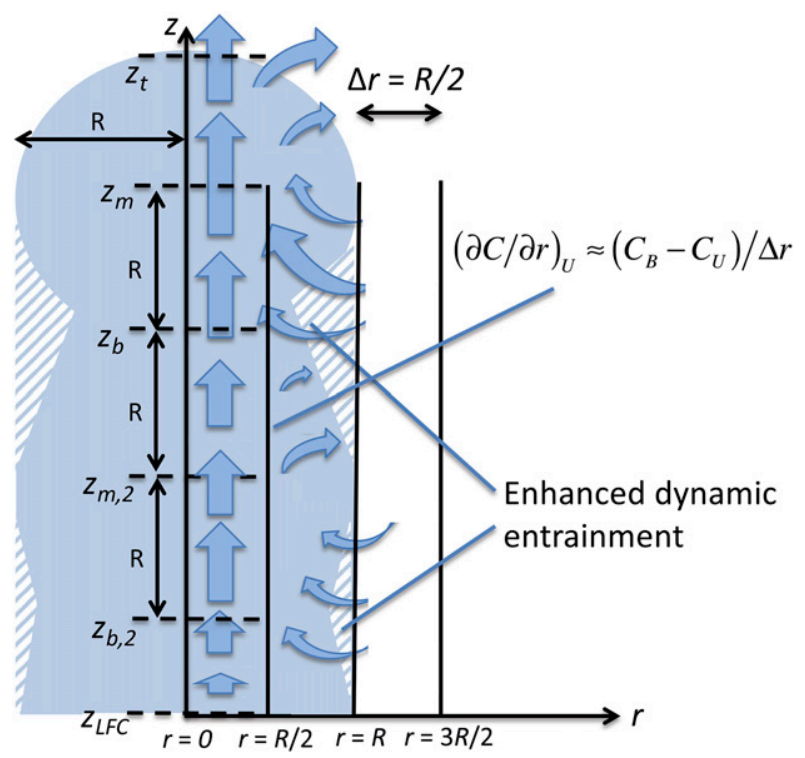

FIG. 4. A diagram of the discretization used for the simple theoretical approximation of thermal chain dynamics. The axisymmetric framework has coordinates in height $z$ and horizontal distance from the vertical axis of symmetry $r . R$ is the updraft radius. $z_{b}, z_{m}$, and $z_{t}$ are the heights of the bottom, middle, and top of the primary ascending thermal, respectively. $z_{m, 2}$ is the height a distance of $R$ below the primary thermal at approximately the center of the second thermal (for conditions in which additional thermals form), and $z_{b, 2}$ is a distance of below the primary thermal at the bottom of the second thermal. $z_{\mathrm{LFC}}$ is the height of the level of free convection. Solid blue shading indicates the cloudy updraft region, i.e., the region containing cloud water and vertical velocities above $\sim 1 \mathrm{~m} \mathrm{~s}^{-1}$.

$$
\left(\frac{D C}{D t}\right)_{R / 2} \approx-\frac{u_{R}\left(C_{R}-C_{R / 2}\right)}{R} .
$$

Next, $u_{R}$ is written in terms of $w$ by integrating the mass continuity equation in axisymmetric coordinates from $r=0$ to $R$ to give

$$
u_{R}=-\frac{R}{2} \frac{\partial \hat{w}}{\partial z}
$$

where $\hat{w}$ is $w$ averaged horizontally from $r=0$ to $R$ in the "absolute" (as opposed to thermal-relative) frame of reference with $w=0$ at the surface. Combining (16) and (17) and using $\partial \hat{w} / \partial z=\alpha \partial w / \partial z$, where $\alpha$ is defined as the ratio of $\hat{w}$ to $w$ at $r=0$ defined in section $2 \mathrm{~b}$ (evaluated at height $z_{b}$ ), gives

$$
\left(\frac{D C}{D t}\right)_{R / 2} \approx \frac{\left(C_{R}-C_{R / 2}\right)}{2} \alpha \frac{\partial w}{\partial z}
$$

Defining $\lambda \equiv W / w_{m}$, we can express the thermal ascent rate $W$ as $\lambda w_{m}$ and write the total derivative as $D / D t=(d z / d t)(D / D z)=$ $\lambda w_{m} D / D z$ (where $D / D z$ is the change of a quantity as a function of the thermal height) on the left-hand side of (18) to yield

$$
\lambda w_{m}\left(\frac{D C}{D z}\right)_{R / 2} \approx \frac{\left(C_{R}-C_{R / 2}\right)}{2} \alpha \frac{\partial w}{\partial z},
$$


To solve (19) we make several simplifying assumptions. The basic idea is to take the simplest possible approach that qualitatively captures flow features without adding extraneous detail incommensurate with these simplifications. Thus, we approximate the total and partial derivatives in (19) with simple linear difference operators so that $(D C / D z)_{R / 2} \approx\left(C_{R / 2}-\right.$ $\left.C_{\mathrm{LFC}}\right) / z_{b}$ and $\partial w / \partial z \approx w_{m} / z_{m}$, where $z_{b}$ is the bottom height of the thermal as it rises over time, $C_{\mathrm{LFC}}$ is the tracer value at $t=0$, and it is assumed that $w=0$ at $z_{\mathrm{LFC}}$ at all times. It is also assumed for simplicity that $\alpha \sim \lambda$. This relationship is exact for $\alpha$ at height $z_{m}$ when the thermal size and shape do not change; thus, in essence we assume that $\alpha$ at height $z_{b}$ is equal to $\alpha$ at height $z_{m}$. Finally, we make an additional simplifying assumption that $z_{b} \sim z_{m}$, which is well justified once thermals rise more than a few radii above the LFC.

Combining these approximations with (19), using $c_{R}=0$ (tracer value of 0 in the environment), and rearranging terms gives $C_{R / 2} \approx(2 / 3) C_{\mathrm{LFC}}$. This is the same expression as Eq. (31) in M2017, but derived in a Lagrangian framework following the primary thermal's ascent. Assuming that lateral turbulent mixing reduces $C_{R / 2}$ and $C_{0}$ (i.e., the value of $C$ at $r=0$ ) by the same relative amount, then

$$
C_{R / 2} \approx \frac{2}{3} C_{0}
$$

Equation (20) suggests an increase in horizontal gradients of $C$ between $r=0$ and $r=R / 2$ by a factor of $3 / 2$ near the thermal bottom, relative to the upper part of the thermal that is not directly affected by dynamic entrainment. Because the horizontal turbulent flux divergence of $C$ is based on a first-order closure method as detailed above, it depends on the product of horizontal gradients of $C$ and $w$. Assuming a similar $3 / 2$ scaling applies to gradients of $w$ for simplicity, even though it is not a passive scalar, this suggests an enhancement of lateral turbulent mixing by a factor of $\xi_{b}=(3 / 2)^{2}=9 / 4$ owing to dynamic entrainment at height $z_{b}$. Following the derivation in section $2 \mathrm{~b}$ except applying the enhanced lateral turbulent mixing at the thermal bottom gives a fractional entrainment rate at $z_{b}$ of

$$
\varepsilon_{b}=2 \frac{\xi_{b} k^{2} L}{P_{r} R^{2}}=\frac{9 k^{2} L}{2 P_{r} R^{2}} .
$$

Qualitatively, enhanced mixing at $z_{b}$ is a general feature and does not depend on the particular finite difference method or other approximations used in the derivation, so long as $\partial w / \partial z>0$ locally which is expected generally because of the primary thermal's toroidal circulation. However, because $\partial w / \partial z$ at height $z_{b}$ is estimated using a simple "central" finite difference between $w$ at heights $z_{m}$ and $z_{\text {LFC }}$, this probably represents a lower bound quantitative estimate of $\partial w / \partial z$; this estimate does not account for enhanced dilution and reduction of $B$ and $w$ at $z_{b}$ relative to $z_{m}$ that may locally increase gradients in $w$ between $z_{b}$ and $z_{m}$. Indeed, as shown in Part II this simple approach tends to underestimate the enhancement of $\varepsilon$ locally at $z_{b}$ compared to numerical simulations, particularly LES. However, including this nonlinear effect would add considerable detail and is counter to our approach of applying the simplest approximations to capture the main physical features.

With the fractional entrainment rate above, we use the same approach as in section $2 \mathrm{~b}$ to derive expressions for $C$ and $B$ at $z_{b}$, noting ${ }^{4}$ that for a coherent thermal $d z_{b} / d t=W=w_{t}$ :

$$
\begin{aligned}
C_{b} & =\frac{1-\frac{\varepsilon_{b} z_{b}}{2}}{1+\frac{\varepsilon_{b} z_{b}}{2}} C_{\mathrm{LFC}}, \\
B_{b} & =\frac{B_{A D}-\varepsilon_{b} \Omega}{1+\frac{\varepsilon_{b} z_{b}}{2}} .
\end{aligned}
$$

For $w$, we similarly integrate the vertical momentum equation from $z_{\text {LFC }}$ to $z_{b}$, neglecting $p_{d}$ forcing for simplicity, using the expression for $p_{b}$ forcing from Morrison (2016a) and the fractional entrainment rate at $z_{b}$ to yield

$$
w_{b}=\sqrt{2\left(1+\frac{2 \alpha^{2} R^{2}}{z_{t}^{2}}\right)^{-1}\left(1+\frac{2}{3} P_{r} \varepsilon_{b} z_{b}\right)^{-1} \int_{z_{\mathrm{LFC}}}^{z_{b}} B_{b} d z_{b}^{*}} .
$$

\section{d. Expressions for $\varepsilon, C, B$, and $w$ below the primary ascending thermal}

Enhanced mixing from dynamic entrainment near the bottom of the primary ascending thermal at height $z_{b}$ can locally reduce $B$ and $w$, thereby decreasing $\partial B / \partial z$ and $\partial w / \partial z$ below $z_{b}$ (compared to if there was no enhanced mixing near $z_{b}$ ) at a height we will call $z_{m, 2}$ (Fig. 4). In this case, dynamic entrainment will be locally reduced at $z_{m, 2}$ because of the decrease in $\partial w / \partial z$. This locally reduced dynamic entrainment in turn can lead to the emergence of a secondary maximum in $w$ at height $z_{m, 2}$, in which case a second thermal and associated toroidal circulation will develop below the primary ascending thermal. From the numerical simulations in Part II (e.g., see Figs. 2 and 14-17 therein), the distance between $z_{b}$ and $z_{m, 2}$ is approximately $R$, for the simulations that have multiple thermals. Thus, we assume that $z_{m, 2}=z_{b}-R=z_{t}-3 R$. For simplicity, $C, B$, and $w$ are all calculated at height $z_{m, 2}$, while $w_{m, 2}$ is obtained by integrating the buoyancy calculated at $z_{m, 2}$ as it ascends. In effect, we neglect the impact of dynamic pressure forcing on $w$ at height $z_{m, 2}$, but the height and magnitude of the secondary $w$ maxima in the numerical simulations in Part II are well estimated by this approach (see Figs. 12 and 13 therein).

Using the same approach as that used to derive the dynamic entrainment enhancement factor at the thermal bottom $\xi_{b}$, we seek to obtain an expression for $\xi$ at height $z_{m, 2}$. We apply the same horizontal discretization that was used to derive (19).

\footnotetext{
${ }^{4}$ The assumption that the ascent rate of a parcel at the bottom of the thermal is equal to that of the thermal as a whole (i.e., $d z_{b} / d t=$ $W$ is not strictly valid because moist thermals are in actuality not closed, fully coherent flow structures-that is, a rising parcel initially at the thermal bottom may subsequently be detrained from the thermal if the parcel's vertical velocity $<W$. Nonetheless, this is assumed for simplicity, and is qualitatively supported by the results in Part II.
} 
Similarly, $\partial \hat{w} / \partial z$ at height $z_{m, 2}$ is estimated using a simple linear "central" difference operator for $w$ between $z_{b}$ and $z_{\mathrm{LFC}}$, where again $w=0$ at $z_{\mathrm{LFC}}$ in the "absolute" reference frame. This gives

$$
\left(\frac{\partial \hat{w}}{\partial z}\right)_{m, 2} \approx \frac{\widehat{w_{b}}}{z_{b}}=\alpha \frac{w_{b}}{z_{b}}
$$

where $w_{b}$ is given by (24). Combining (25) and (19) gives

$$
W\left(\frac{D C}{D z}\right)_{R / 2}=\lambda w_{m}\left(\frac{D C}{D z}\right)_{R / 2} \approx \frac{\left(C_{R}-C_{R / 2}\right)}{2} \frac{\alpha w_{b}}{z_{b}},
$$

where $W$ is the ascent rate of the primary thermal because height $z_{m, 2}$ is assumed to be at a fixed distance of $R$ below the primary thermal as it ascends.

As above at height $z_{b}$, we approximate the total derivative $D / D z$ using a simple linear difference operator so that $(D C / D z)_{R / 2} \approx$ $\left(C_{R / 2}-C_{\mathrm{LFC}}\right) / z_{m, 2}$ and take $\alpha \sim \lambda$. Using these approximations in (26), setting $C_{R}=0$, and rearranging terms gives

$$
C_{R / 2} \approx\left(1+\frac{w_{b} z_{m, 2}}{2 w_{m} z_{b}}\right)^{-1} C_{\mathrm{LFC}}
$$

Based on the same argument as above for mixing at $z_{b}$, (27) suggests a scaling factor of $\xi_{m, 2}=\left[1+w_{b} z_{m, 2} /\left(2 w_{m} z_{b}\right)\right]^{2}$ for lateral turbulent mixing at height $z_{m, 2}$. Applying this scaling gives expressions for $\varepsilon, C, B$, and $w$ at $z_{m, 2}$ following (21)-(24), except that $\xi_{b}$ is replaced by $\xi_{m, 2}, z_{b}$ by $z_{m, 2}$, and the vertical integral of buoyancy is from $z_{\mathrm{LFC}}$ to $z_{m, 2}$.

As $w_{b}$ becomes relatively large, $\xi_{m, 2}$ increases. This makes physical sense, because if $w_{b}$ is relatively large, this implies a positive vertical gradient in $w$ below $z_{b}$ and hence substantial dynamic entrainment at $z_{m, 2}$. We therefore expect an entrainment enhancement factor greater than 1, comparable to that derived at height $z_{b}\left(\xi_{b}=9 / 4\right)$ as $w_{b}$ approaches $w_{m}$. Conversely, as $w_{b} \rightarrow 0, \xi_{m, 2} \rightarrow 1$. This makes intuitive sense, because a vanishing $w$ at the base of the primary ascending thermal implies a weak or negative vertical gradient in $w$ below the primary thermal and hence little or no dynamic entrainment at height $z_{m, 2}$. Thus, a sufficiently small $w_{b}$ from enhanced dilution at the thermal bottom is critical to the existence of multiple local maxima in $w$ (indicative of the thermal chain structure).

This approach can be extended to derive expressions for $\varepsilon, C$, $B$, and $w$ at the bottom of the potential second thermal at height $z_{b, 2}$, where $z_{b, 2}=z_{m, 2}-R$. This is done by again approximating the average $\partial \hat{w} / \partial z \approx \alpha w_{m, 2} / z_{m, 2}$ at the bottom of the second thermal using a simple linear difference operator. This gives an equation similar to (27), except $w_{b} z_{m, 2} /\left(2 w_{m} z_{b}\right)$ is replaced by $w_{m, 2} z_{b, 2} /\left(2 w_{m} z_{m, 2}\right)$. The corresponding enhancement factor for turbulent mixing is then $\xi_{b, 2}=\left[1+w_{m, 2} z_{b, 2} /\left(2 w_{m} z_{m, 2}\right)\right]^{2}$. Expressions for $\varepsilon_{b, 2}, C_{b, 2}, B_{b, 2}$, and $w_{b, 2}$ follow from (21)-(24), except that $\xi_{b}$ is replaced with $\xi_{b, 2}, z_{b}$ by $z_{b, 2}$, and buoyancy is integrated from $z_{\mathrm{LFC}}$ to $z_{b, 2}$ in the $w$ equation.

Using the same approach, we derive a general expression $\xi_{m, i}=\left[1+w_{b, i} z_{m, i} /\left(2 w_{m} z_{b, i}\right)\right]^{2}$ for the entrainment enhancement factor below the potential secondary thermal, where $z_{m, i}$ lies a distance of $R$ below $z_{b, i}$. Similarly, a general expression $\xi_{b, i}=\left[1+w_{m, i-1} z_{b, i} /\left(2 w_{m} z_{m, i-1}\right)\right]^{2}$ is derived for the enhancement factor at height $z_{b, i}=z_{m, i-1}-R$, i.e., at the bottom of potential additional thermals. Correspondingly, general expressions for $\varepsilon, C, B$, and $w$ for any $i$ are obtained from (21)-(24) by replacing $\xi_{b}$ with $\xi_{m, i}$ or $\xi_{b, i}, z_{b}$ with $z_{m, i}$ or $z_{b, i}$, and integrating buoyancy from $z_{\mathrm{LFC}}$ to $z_{m, i}$ or $z_{b, i}$ in the $w$ equation.

\section{Results from the theoretical expressions for $C, B$, and $\boldsymbol{w}$}

Results from the theoretical expressions derived in section 2 for evolution of a passive scalar $C$, buoyancy $B$, and vertical velocity $w$ are presented in this section. In these calculations, the thermodynamic sounding is from Weisman and Klemp (1982) with a low-level water vapor mixing ratio of $14 \mathrm{~g} \mathrm{~kg}^{-1}$. The $R_{H}$ of the original Weisman and Klemp (1982) sounding is modified above $1.5 \mathrm{~km}$ to have a constant value of 0.425 or 0.85 . The turbulent mixing length $L$ is set equal to $R / 3$. This scaling is somewhat ad hoc, but is based on the idea that the scale of eddies driving turbulent mixing is related to $R$, with $R$ corresponding to the peak in the $w$ kinetic energy spectrum (Lebo and Morrison 2015; Peters et al. 2019) and nearly isotropic turbulence within an inertial subrange at scales smaller than some fraction of $R$. The mixing parameter $k^{2}=0.18$ and the Prandtl number $\operatorname{Pr}=1 / 3$ following Deardorff (1972), and we use $\alpha=0.5$.

Note, variations in the sounding $R_{H}$ have two distinct effects. First, the environmental $R_{H}$ directly affects the properties of entrained air and hence evolution of $B$ and $w$. Second, the environmental $R_{H}$ directly affects air density of the background environment, and as a consequence alters the environmental virtual temperature. Because of this effect, CAPE is $5 \%$ larger when $R_{H}=0.425$ than when $R_{H}=0.85$. To separate these effects clearly, the adiabatic buoyancy $B_{\mathrm{AD}}$ in the expressions for $B$ and $w$ is based on the virtual temperature from the original WK sounding (using the $R_{H}$ from that sounding), and is the same in both the $R_{H}=0.425$ and $R_{H}=0.85$ cases. Since the CAPE in the $R_{H}=0.85$ and original formulations of the WK82 sounding are nearly identical, the aforementioned approximation for $B_{\mathrm{AD}}$ is approximately $5 \%$ too small when $R_{H}=0.425$. Finally, we apply the conditions that $C=1 \mathrm{~kg} \mathrm{~kg}^{-1}$ and $B$ and $w$ are equal to zero at the LFC $(\sim 1400 \mathrm{~m}$ for the sounding, in the coordinate system with $z=0$ at the surface). For $w$, the buoyancy term is vertically integrated using a simple Euler forward method and $100 \mathrm{~m}$ vertical spacing. Our analysis focuses on updrafts with a top below $8 \mathrm{~km}$. This is because the simulations in Part II using the same thermodynamic soundings show a rapid increase in $R$ above $\sim 7-8 \mathrm{~km}$ and generation of considerable negative buoyancy within thermals reaching this height, contrasting with assumptions used in deriving the theoretical expressions.

Vertical profiles of $C, B$, and $w$ are shown in Fig. 5 for the $R_{H}=0.425$ cases and in Fig. 6 for $R_{H}=0.85$. $R$ ranges from 630 to $1900 \mathrm{~m}$ in both figures. Note that for wide updrafts, $w$ can locally exceed the thermodynamic maximum equal to $\sqrt{2 \int B_{\mathrm{AD}} d z}$ (Figs. 51 and 6i,l), where $\int B_{\mathrm{AD}} d z$ is the adiabatic buoyancy integrated from the LFC up to the given height on the $y$ axis. This occurs because of the effects of dynamic pressure forcing at $z_{m}$ that are considered in the derivation of 

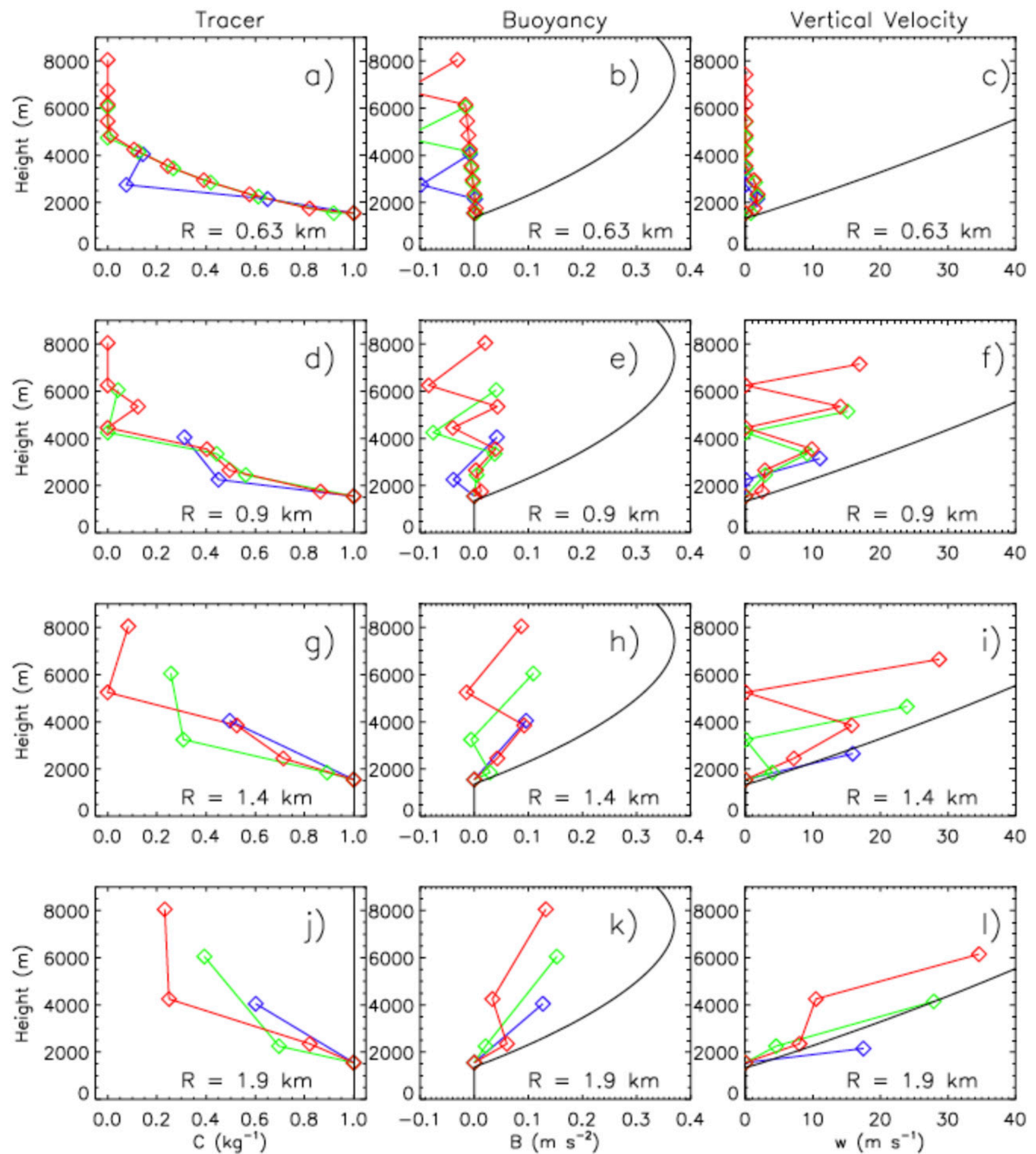

FIG. 5. Vertical profiles of (left) scalar mixing ratio $C$, (center) buoyancy $B$, and (right) vertical velocity $w$ from the theoretical expressions. Blue, green, and red lines show results for updrafts with tops of 4,6 , and $8 \mathrm{~km}$, respectively. Black lines in the left and center columns show the undilute $C$ and $B$, respectively, and in the right column show the thermodynamic maximum $w$ given by $\sqrt{2 \int B_{\mathrm{AD}} d z}$, where $\int B_{\mathrm{AD}} d z$ is calculated by integrating adiabatic buoyancy up to a given height on the $y$ axis. In all plots the diamond symbols show values from the theoretical expressions at the heights where the expressions are calculated, with straight lines connecting these points for illustration. Results are shown for environmental relative humidity $R_{H}=0.425$ and various updraft radii $R$ as indicated. 

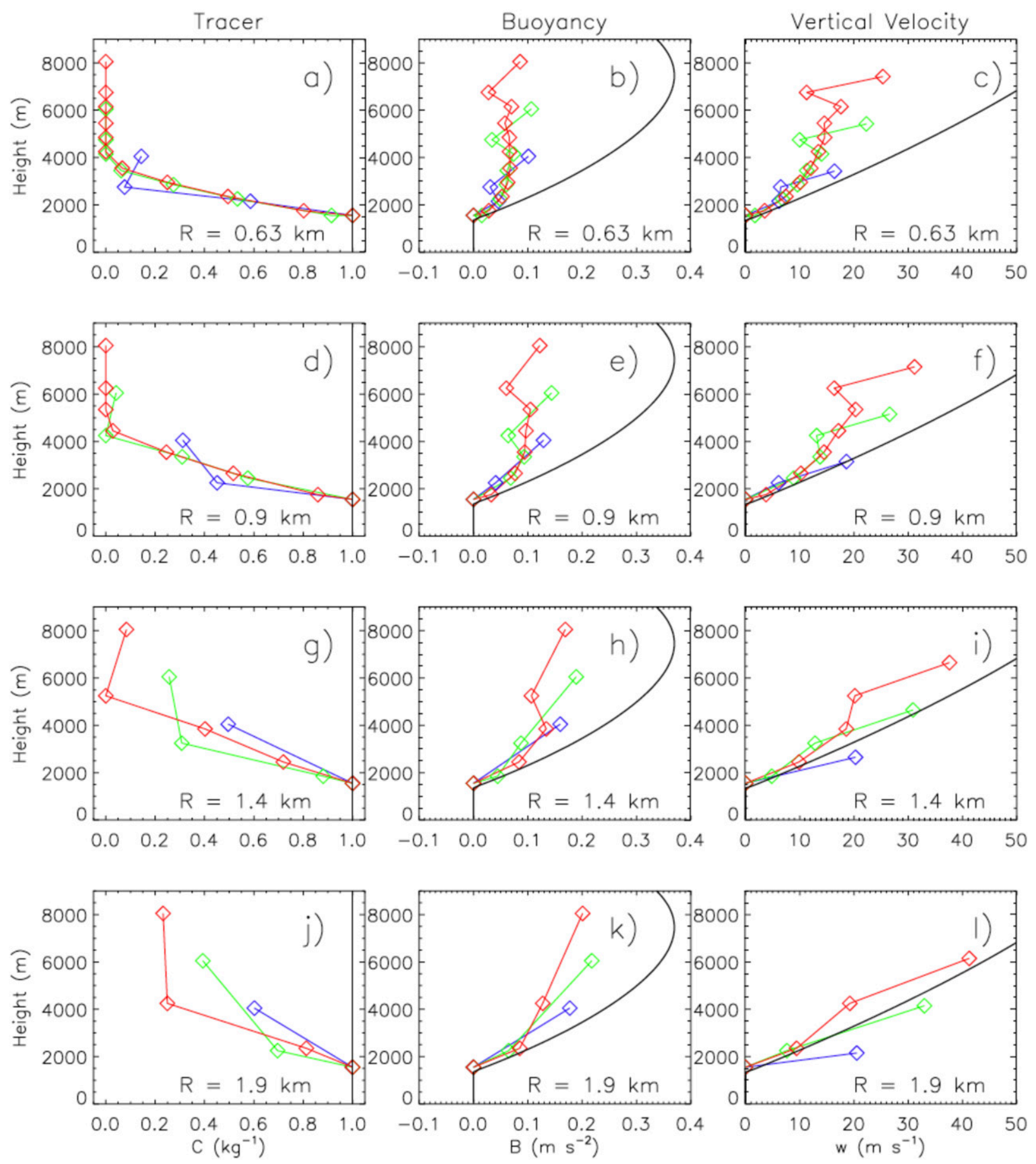

FIG. 6. As in Fig. 5, but for environmental relative humidity $R_{H}=0.85$.

$w_{m}$ (see section $2 \mathrm{~b}$ ). The effects of mixing and dilution are readily apparent by the rapid decrease of $C$ with height from the values of $1 \mathrm{~kg}^{-1}$ at the LFC (Figs. 5a,d,g,j and 6a,d,g,j). Although dilution decreases as $R$ is increased, there is still considerable dilution even for the largest $R$ shown $(1900 \mathrm{~m})$; values are about $0.25 \mathrm{~kg}^{-1}$ at top of the primary ascending thermal when it reaches $8 \mathrm{~km}$ for both the dry and moist cases.
Increased mixing at the bottom of the thermals relative to the top is seen in the $C$ profiles for most cases, highlighting the effects of locally enhanced entrainment and dilution. Critically, the effects of increased dilution at the thermal bottoms are much greater for $B$ and $w$ than $C$, as seen by the sharp $B$ and $w$ local minima in many cases, particularly for the dry environment. 
The theoretical $w$ are further illustrated in Fig. 7, which shows $w$ at the center of the primary thermal $\left(z_{m}\right)$, the bottom of the primary thermal $\left(z_{b}\right)$, and below the primary thermal $\left(z_{m, 2}\right)$ as a function of the primary thermal top height as it ascends. For relatively dry conditions and small $R$, updrafts occur as isolated rising thermals with only one thermal present through the depth of the troposphere at a given time. This is seen in Fig. 7a for $R_{H}=0.425$ and $R=0.63 \mathrm{~km}$ by red and blue lines that do not overlap anywhere in the vertical (except for zero values), meaning that only a single thermal with nonzero $w$ is present at a given time. This $w$ structure occurs because of the rapid reduction of buoyancy from mixing of updraft and environmental air below the primary ascending thermal when $R$ and $R_{H}$ are small. The theoretical expressions do not account for secondary thermals rising into a negatively buoyant wake of the primary thermal when the environment is dry, which may further limit the generation and ascent of secondary thermals. In contrast, for wide updrafts the structure resembles a starting plume (Turner 1962), with the primary thermal at the head of the ascending updraft but no distinct second thermal (Figs. $7 \mathrm{f}-$ h). In other words, there is no local secondary maximum of $w$ below the primary thermal. In these cases there is limited local reduction of $B$ and $w$ even with enhanced mixing at the bottom of the primary ascending thermal.

Multiple thermals indicative of a thermal chain structure occur for $R$ of 0.9 or $1.4 \mathrm{~km}$ when $R_{H}=0.425$. In these cases buoyancy is reduced considerably at the bottom of the primary ascending thermal from the enhanced mixing at this height, leading to the distinct minima in $w$ (Figs. 5f,i). This structure is much less apparent for the moist environment, with only weak local minima in $B$ and $w$ for $R$ of 0.63 and $0.9 \mathrm{~km}$ (Figs. $6 \mathrm{c}, \mathrm{f}$ ). Overall, the theoretical expressions suggest structural transitions for growing cumulus updrafts from an isolated rising thermal to a thermal chain to a starting plume as nondimensional updraft radius $R^{2} /(z L)$ and/or $R_{H}$ are increased. For a dry environment and small $R^{2} /(z L)$, the structure is in line with the traditional rising thermal conceptual model. For a moist environment and/or large $R^{2} /(z L)$, the structure resembles a rising plume with only a single $w$ maximum. The thermal chain represents a transition between these thermal and starting plume regimes. Note that because dilution scales with the nondimensional radius $R^{2} /(z L)$, for deeper updrafts than those analyzed here, a thermal chain structure emerges even for large $R>1.4 \mathrm{~km}$ in a dry environment. To assess the sensitivity of these results to adiabatic buoyancy, the theoretical expression was reevaluated with $B_{\mathrm{AD}}$ reduced by a factor of 2 at all levels, equivalent to a factor of 2 reduction in CAPE (Fig. 8). With the decrease in CAPE, structural transitions from isolated thermal to thermal chain to starting plume are shifted to larger $R$ for both dry and moist environments. For example, there is a distinctive thermal chain structure for the moist environment and $R=0.63 \mathrm{~km}$ (Fig. $8 \mathrm{~b}$ ), in contrast to the results with larger CAPE; $w$ is zero at the bottom of the primary ascending thermal but with local maxima of $\sim 5$ to $10 \mathrm{~m} \mathrm{~s}^{-1}$ both above and below after the updraft top rises above $\sim 4 \mathrm{~km}$.

The role of various forcing mechanisms on $w$ is investigated by omitting certain terms in the expressions for $w$ from section 2 (Fig. 9). The $w$ profiles are calculated by (i) neglecting the pressure term, (ii) neglecting the mixing of $B$ (including dry air entrainment), (iii) allowing mixing of $B$ but neglecting dry air entrainment, and (iv) neglecting momentum mixing. For $R \leq 1.5 \mathrm{~km}$, the pressure term has little effect (not shown). Mixing and dilution of $B$ is the key mechanism reducing $w$ from the thermodynamic maximum value $\left(\sqrt{2 \int B_{\mathrm{AD}} d z}\right)$, particularly in the drier environment. In this environment, most of the reduction of $B$ and hence $w$ from dilution is from mixing of dry environmental air and associated evaporation and/or reduction in condensation rates. The direct dilution of buoyancy (i.e., isobaric mixing of $T$ between the updraft and environment) is also important. Overall, momentum mixing has a fairly limited impact, especially for the dry environment, as seen by the closeness of the orange and dark blue lines in Fig. 9. Interestingly, the effects of dry air mixing on $w$ are similar to the effects of momentum mixing in the humid environment, seen by the closeness of the orange and red lines in Figs. 9c and 9d. The effects of momentum mixing are relatively larger for the high $R_{H}$ cases than the low $R_{H}$ cases because momentum mixing scales with $w^{2}$, and it is generally larger in the humid environment for a given $R$.

The critical impact of dry-air mixing in low- $R_{H}$ environments on condensation/evaporation and hence $B$ and $w$ is consistent with the relatively weak response of $C$ to enhanced mixing from dynamic entrainment at the thermal bottoms compared to $B$ and $w$ (Fig. 5). These results indicate the important role of dry environmental-air engulfment and locally enhanced mixing near the thermal bottoms for driving the distinctive thermal chain structure with sharp local maxima and minima of $B$ and $w$. This is confirmed by calculations analogous to those shown in Figs. 5 and 6, except the contribution from mixing of updraft and $d r y$ environmental air is neglected entirely (equivalent to assuming $R_{H}=1$ in the expressions for $B$ and $w$ ). Note in these tests there is still buoyancy dilution from isobaric mixing of environment and updraft temperatures. Although there are local $w$ minima and maxima within the profile, differences between the minima and maxima are small (Fig. 10). This occurs even when $R$ is very small (e.g., 300 m; Figs. 10a,b). Thus, we propose that the distinctive thermal chain structure with sharp peaks in $w$ and $B$ is primarily a feature of moist convection, owing mainly to the effects of dry-air entrainment.

\section{Discussion and implications for fractional entrainment rates}

Results from the theoretical expressions suggest the importance of enhanced entrainment associated with pulses of inflowing dry environmental air near the bottom of individual thermals on overall updraft behavior. For multiple thermals in a chain, this implies a complicated structure of fractional entrainment rate $\varepsilon$. Before proceeding, we wish to clearly distinguish between entrainment and dilution. The $\varepsilon$ derived in section 2 is based on a bulk approach that essentially assumes a 1:1 relationship between dilution of a conserved variable at $r=$ 0 (the updraft's vertical axis of symmetry) and $\varepsilon$. However, direct calculations of entrainment and detrainment from LES (e.g., Romps 2010; Dawe and Austin 2011b) differ from bulk estimates because of variations in the environment of the conserved variable field used to calculate $\varepsilon$ that are neglected 

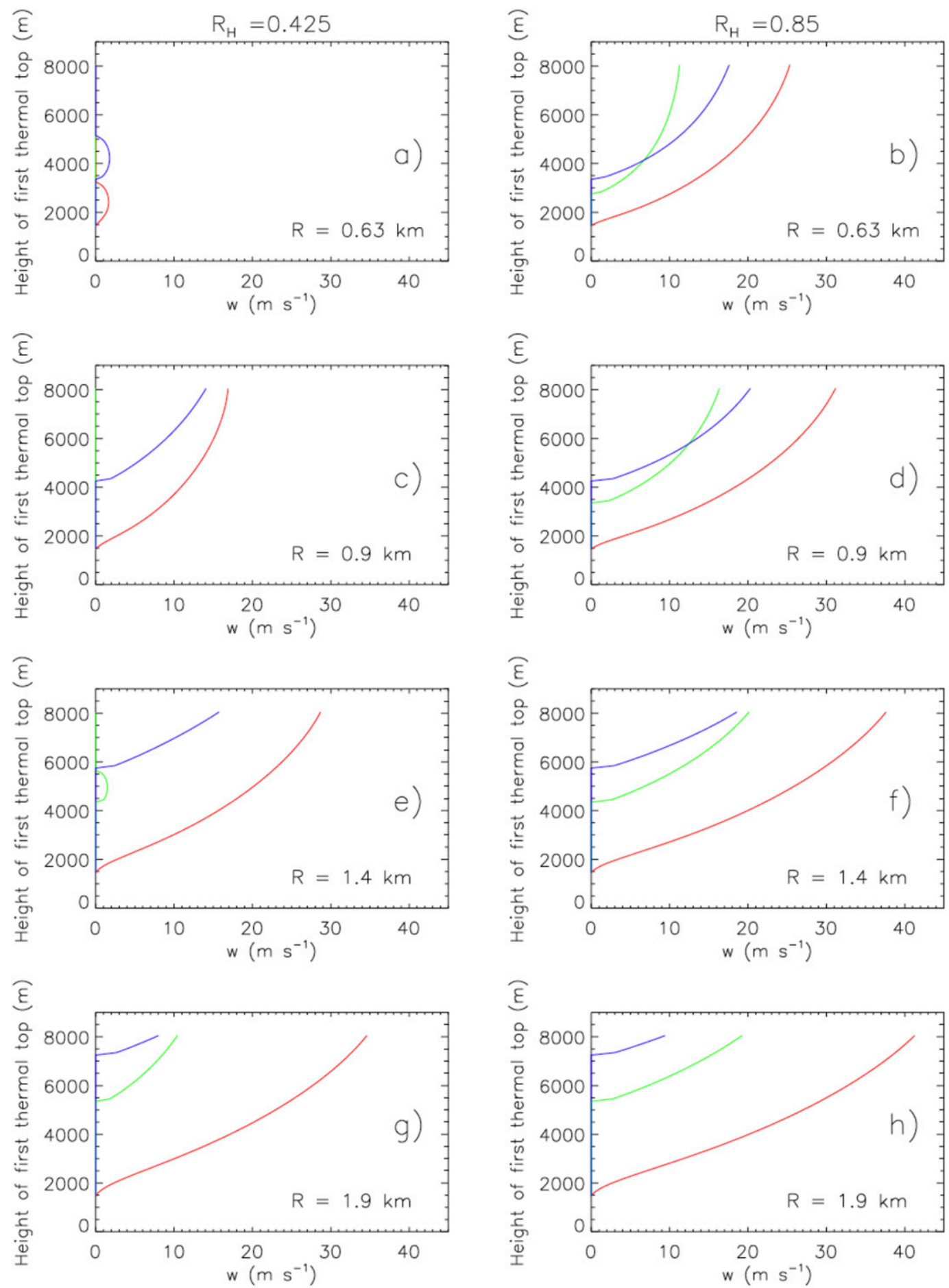

FIG. 7. Evolution of vertical velocity $w$ for ascending updraft thermals calculated from the theoretical expressions. The red line is $w$ at the center of the primary ascending thermal at height $z_{m}$. The green line is $w$ at the bottom of the first thermal at height $z_{b}$, and the blue line is $w$ at height $z_{m, 2}$, a distance of $R$ below $z_{b}$. Moving horizontally within each plot shows $w$ at these three locations when the updraft top is at the height indicated along the $y$ axis. Thus, $z_{b}$ and $z_{m, 2}$ are located below this height. Note that in (a) the uppermost thermal completely decays before the development of the second thermal; in this case the height of the second thermal is still plotted with respect to height of where the first thermal would be had it not decayed based on the assumed separation distance between the center of the second thermal (height $\left.z_{m, 2}\right)$ and top of the first thermal $\left(z_{t}\right)$ of $3 R$ (see Fig. 4). Because the thermals rise over time the $y$ axes also correspond to time, with the height-to-time translation depending on the ascent rate of the first thermal. Results are shown with environmental $R_{H}$ of (left) 0.425 and (right) 0.85 . The updraft radius $R$ is indicated in each panel. 

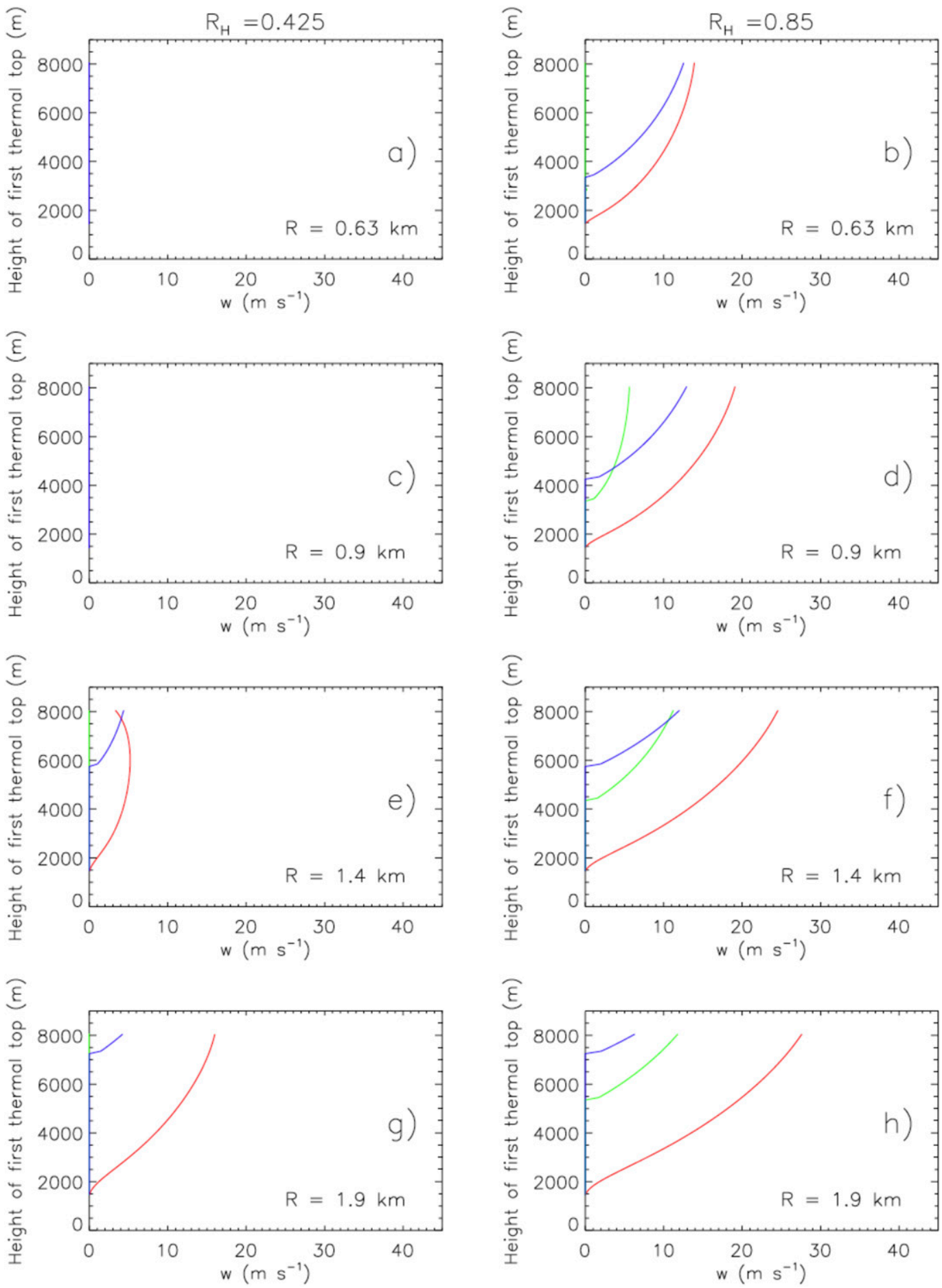

FIG. 8. As in Fig. 7, but for the Weisman and Klemp (1982) thermodynamic sounding with the undilute buoyancy reduced by a factor of 2 .

in bulk estimates. Because of mixing, values in the environment close to the updraft are generally closer to updraft values than those in the far environment. As a result, bulk estimates of $\varepsilon$ tend to be smaller by about a factor of 2 than the direct calculations. Nonetheless, the shape of the bulk and explicit entrainment profiles are quite similar, suggesting the same overall behavior applies to both bulk and explicit entrainment rates (e.g., Romps 2010; Dawe and Austin 2011b).

The complicated behavior of $\varepsilon$ from the theory is illustrated in Figs. 11 and 12 . These figures show vertical profiles of $\varepsilon$ for 

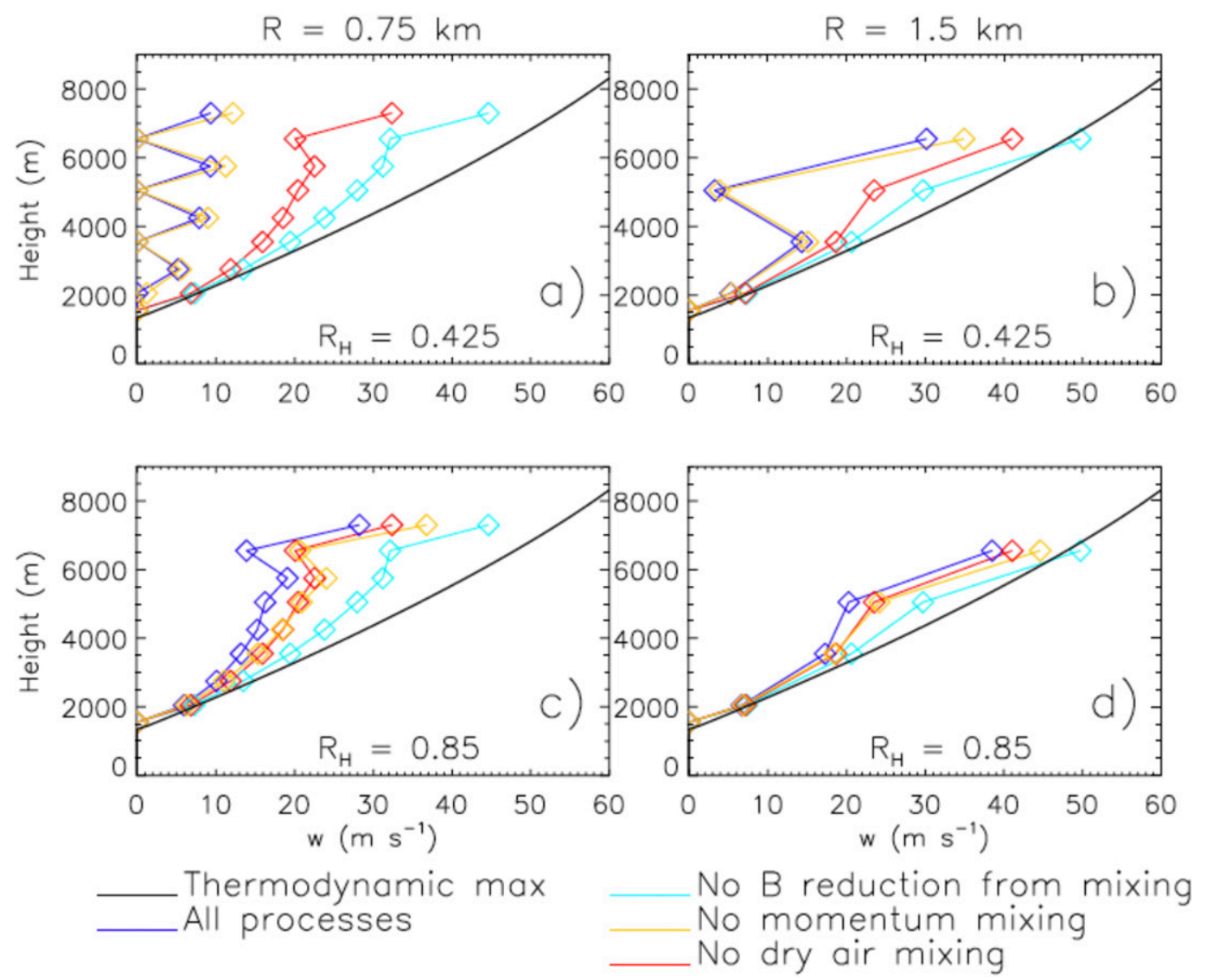

FIG. 9. Vertical profiles of vertical velocity $w$. Colored lines show results with various forcing terms neglected in the theoretical expressions, as labeled below the plots. Results are shown when the primary ascending thermal top is at a height of $8 \mathrm{~km}$. Black lines show the thermodynamic maximum $w$ given by $\sqrt{2 \int B_{\mathrm{AD}} d z}$, where $\int B_{\mathrm{AD}} d z$ is calculated by integrating adiabatic buoyancy up to a given height on the $y$ axis. In all plots the diamond symbols show values from the theoretical expressions at the heights where the expressions are calculated, with lines connecting these points for illustration. Results are shown for updraft radius $R=$ (a), (c) 0.75 and (b),(d) $1.5 \mathrm{~km}$. The environmental relative humidity $R_{H}=$ (top) 0.425 and (bottom) 0.85 .

various heights of the primary ascending thermal top, $R$ of 0.75 and $1.5 \mathrm{~km}$, and $R_{H}$ of 0.425 (Fig. 11) and 0.85 (Fig. 12). The same thermodynamic sounding and values for $k, P_{r}$, and $L$ from section 3 are used to calculate the theoretical $\varepsilon$ shown in the figures. Overall, values of $\varepsilon \sim 0.2$ to $2 \mathrm{~km}^{-1}$ are consistent with previous direct and bulk estimates from LES (e.g., Romps 2010; De Rooy et al. 2013; Dawe and Austin 2011b; Hannah 2017; Zhang et al. 2016; Moser and Lasher-Trapp 2017) and observations (e.g., Lu et al. 2016). Mixing at the bottom of the primary ascending thermal and below is determined by the flow-dependent, nondimensional scaling parameter $\xi$ (see sections $2 c$ and 2 d) which varies between 1 and 9/4, indicated by the triangles in Figs. 11 and 12. The large local increase of $\varepsilon$ at the bottom of individual thermals is clearly seen, particularly for the primary ascending thermal, and follows from the scalings proposed in section 2 . This behavior is in agreement with Moser and Lasher-Trapp (2017), who analyzed entrainment in LES of chain-like cumulus convective thermals using a "direct" calculation similar to the method of Dawe and Austin (2011a). They found sharp increases in $\varepsilon$ near the base of individual thermals associated with their toroidal circulations. Moreover, the magnitude of these local increases, relative to $\varepsilon$ elsewhere, was approximately a factor of 2-3 (see Fig. 6 in Moser and Lasher-Trapp 2017), consistent with our theoretical 9/4 scaling of $\varepsilon_{b}$. Locally enhanced $\varepsilon$ near the bottom of individual thermals is also consistent with the simulations detailed in Part II.

Overall, the magnitude of $\varepsilon$ is similar between the dry and moist environments for a given $R$ (cf. Figs. 11 and 12). Thus, large differences in how $B$ and $w$ evolve as a function of $R_{H}$ are attributable mainly to differences in the properties of entrained air, rather than differences in $\varepsilon$. The dry environment does exhibit sharper maxima and minima associated with additional thermals below the primary thermal than in the moist environment, but for a given $R$ the vertically averaged $\varepsilon$ values are similar. This also explains the lack of correlation between $\varepsilon$ and $R_{H}$ noted below.

It has been previously proposed that $\varepsilon$ is a function of $w^{-1}$ (e.g., Neggers et al. 2002; Anber et al. 2019; Zhang et al. 2016), $B / w^{2}$ (e.g., Gregory 2001; Del Genio and Wu 2010), $z^{-1}$ 

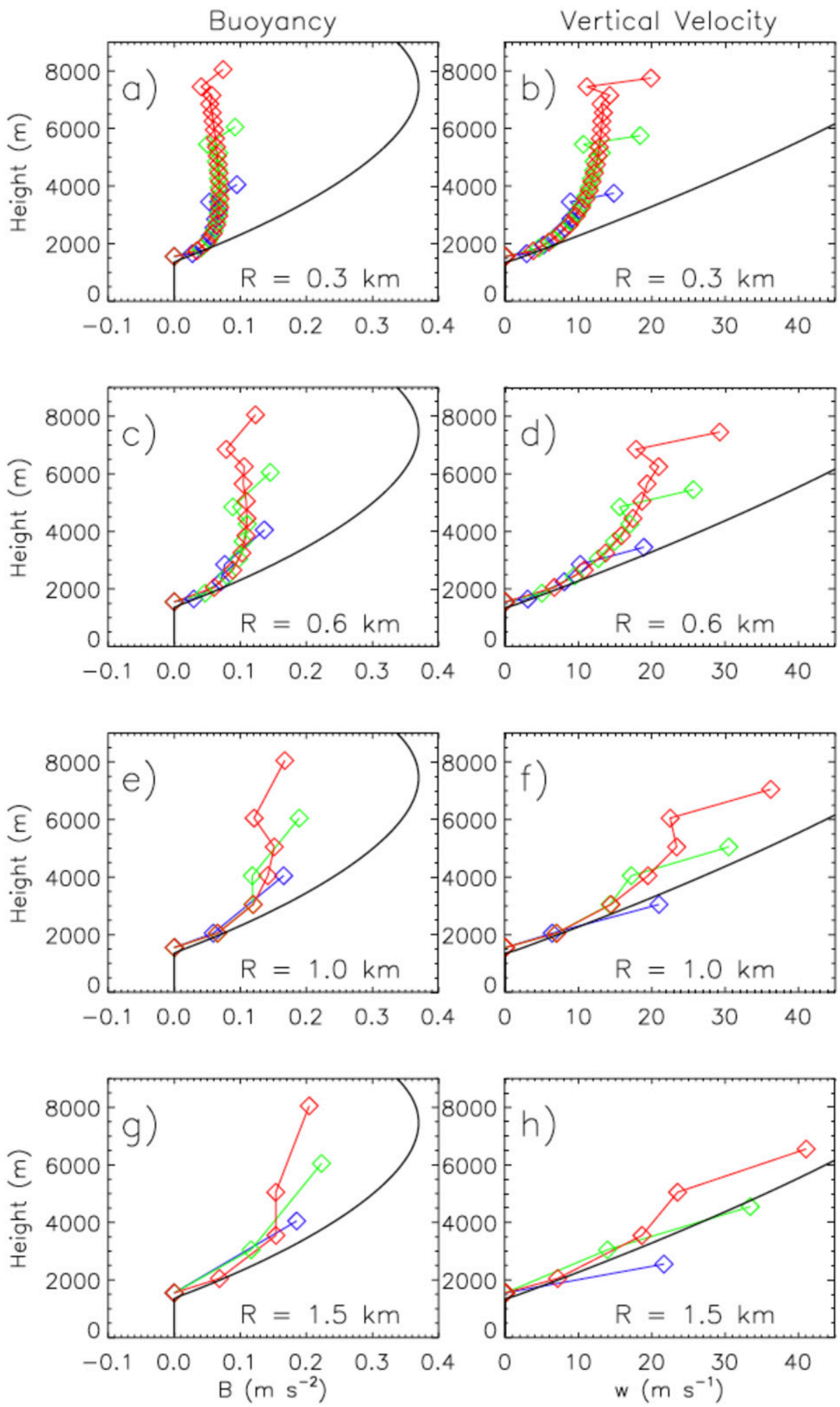

FIG. 10. Vertical profiles of (left) buoyancy $B$ and (right) vertical velocity $w$ from the theoretical expressions with the effects of dry-air entrainment neglected (equivalent to specifying environmental $R_{H}=1$ ). Blue, green, and red lines show results for updrafts with tops of 4,6 , and $8 \mathrm{~km}$, respectively. Black lines in the left column show the adiabatic $B$ and in the right column show the thermodynamic maximum $w$ given by $\sqrt{2 \int B_{\mathrm{AD}} d z}$, where $\int B_{\mathrm{AD}} d z$ is calculated by integrating adiabatic buoyancy up to a given height on the $y$ axis. In all plots the diamond symbols show values from the theoretical expressions at the heights where the expressions are calculated, with lines connecting these points for illustration. Results are shown for various updraft radii $R$ as indicated. 


\section{Fractional Entrainment Rate/ \\ Entrainment Enhancement Factor Vertical Velocity}
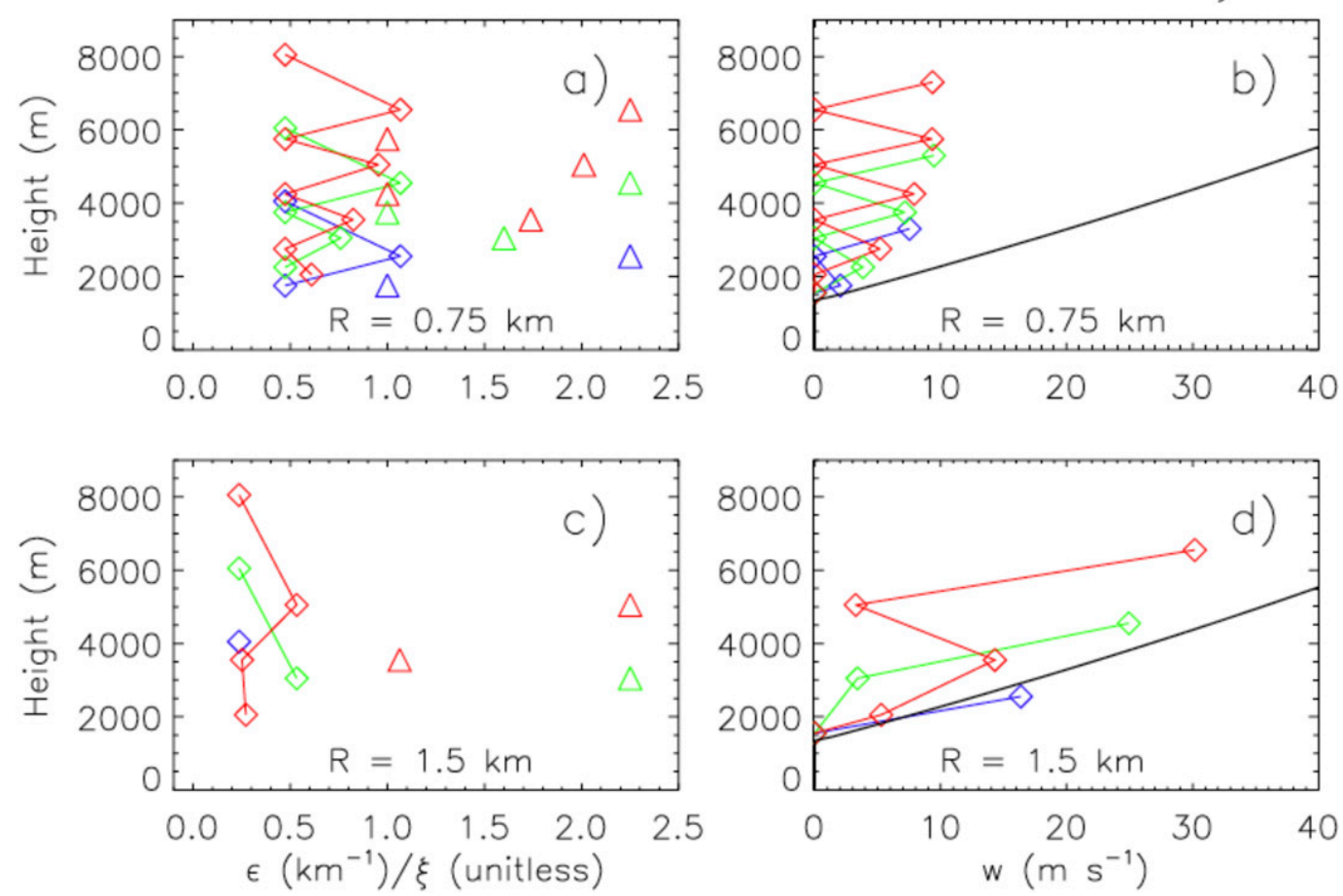

FIG. 11. (a),(c) Vertical profiles of bulk fractional entrainment rate $\varepsilon$ (diamonds and lines) and entrainment enhancement factor $\xi$ (triangles) from the theoretical expressions for the dry environment with $R_{H}=0.425$. Blue, green, and red lines show results for updrafts with tops of 4,6 , and $8 \mathrm{~km}$, respectively. (b),(d) As in (a) and (c), but for the vertical velocity $w$. The black line shows the thermodynamic maximum $w$ given by $\sqrt{2 \int B_{\mathrm{AD}} d z}$, where $\int B_{\mathrm{AD}} d z$ is calculated by integrating adiabatic buoyancy up to a given height on the $y$ axis. Updraft radius values $R$ are (top) 0.75 and (bottom) $1.5 \mathrm{~km}$. In all plots the diamond symbols show values from the theoretical expressions at the heights where the expressions are calculated, with lines connecting these points for illustration.

(e.g., Jakob and Siebesma 2003), $R_{H}$ (e.g., Bechtold et al. 2008), and $R^{-1}$ (e.g., Simpson and Wiggert 1969), or combinations of parameters including $w, B$, and dissipation rate (Lu et al. 2016). Figure 13 shows scatterplots of the theoretical $\varepsilon$ values with $w$, $B, B / w^{2}, z, R_{H}$, and $R^{-1}$. These plots were created by first randomly sampling 1000 points with environmental $R_{H}$ ranging from $15 \%$ to $95 \%$ and $R$ from 150 to $2400 \mathrm{~m}$. For each of the 1000 points, 20 updraft top heights from the LFC to $8 \mathrm{~km}$ were randomly selected to give 20000 sample updrafts. For each sample updraft the expressions for $\varepsilon, B$, and $w$ were evaluated at the heights they are valid (e.g., $z_{t}, z_{m}, z_{b}, z_{m, 2}, z_{b, 2}$ ). Finally, five heights between the LFC and $8 \mathrm{~km}$ were then randomly sampled for each of the 20000 updrafts, with $\varepsilon, B$, and $w$ at the selected heights obtained by linear interpolation from the heights at which the expressions were calculated. If a randomly selected height was above the updraft top, this point was discarded.

Overall, the theoretical expressions produce relationships between $\varepsilon$ and other convective properties similar to those found in recent LES studies (e.g., Hernandez-Deckers and Sherwood 2018). Correlation of the theoretical $\varepsilon$ with many of the convective parameters is poor. There is virtually no correlation with $R_{H}, z^{-1}$, or $B / w^{2}$ (linear correlation coefficient $r_{\text {corr }}$ of $0.082,-0.089$, and -0.005 , respectively), weakly negative correlation of $\varepsilon$ with $w\left(r_{\text {corr }}=-0.205\right)$, and moderately negative correlation with $B\left(r_{\text {corr }}=-0.506\right)$. These negative correlations occur simply because larger fractional entrainment rates lead directly to dilution and reduction of $B$ and vertical velocity. There is a strong positive correlation of $\varepsilon$ with $R^{-1}\left(r_{\text {corr }}=0.918\right)$, which is not surprising as $R^{-1}$ appears directly in the expressions for $\varepsilon$. Nonetheless, even for a given $R$ there is a factor of $\sim 2$ spread of $\varepsilon$ associated with enhanced mixing below the primary thermal.

The strongest relationship from the theoretical expressions is between $\varepsilon$ and $R^{-1}$. An inverse relationship between $\varepsilon$ and $R$ was previously proposed to explain entrainment behavior in several cloud model and LES studies (e.g., Khairoutdinov and Randall 2006; Kirshbaum and Grant 2012; Schlemmer and Hohenegger 2014; Rousseau-Rizzi et al. 2017; HernandezDeckers and Sherwood 2018). Although Hernandez-Deckers and Sherwood (2018) found that $R^{-1}$ explained the most $\varepsilon$ variance of all the quantities they analyzed from their LES, it only accounted for $21 \%$ of the variance. The relatively weak relationship in Hernandez-Deckers and Sherwood (2018) is likely a result of explicitly simulating turbulent updraft flow. 
Fractional Entrainment Rate/

Entrainment Enhancement Factor Vertical Velocity
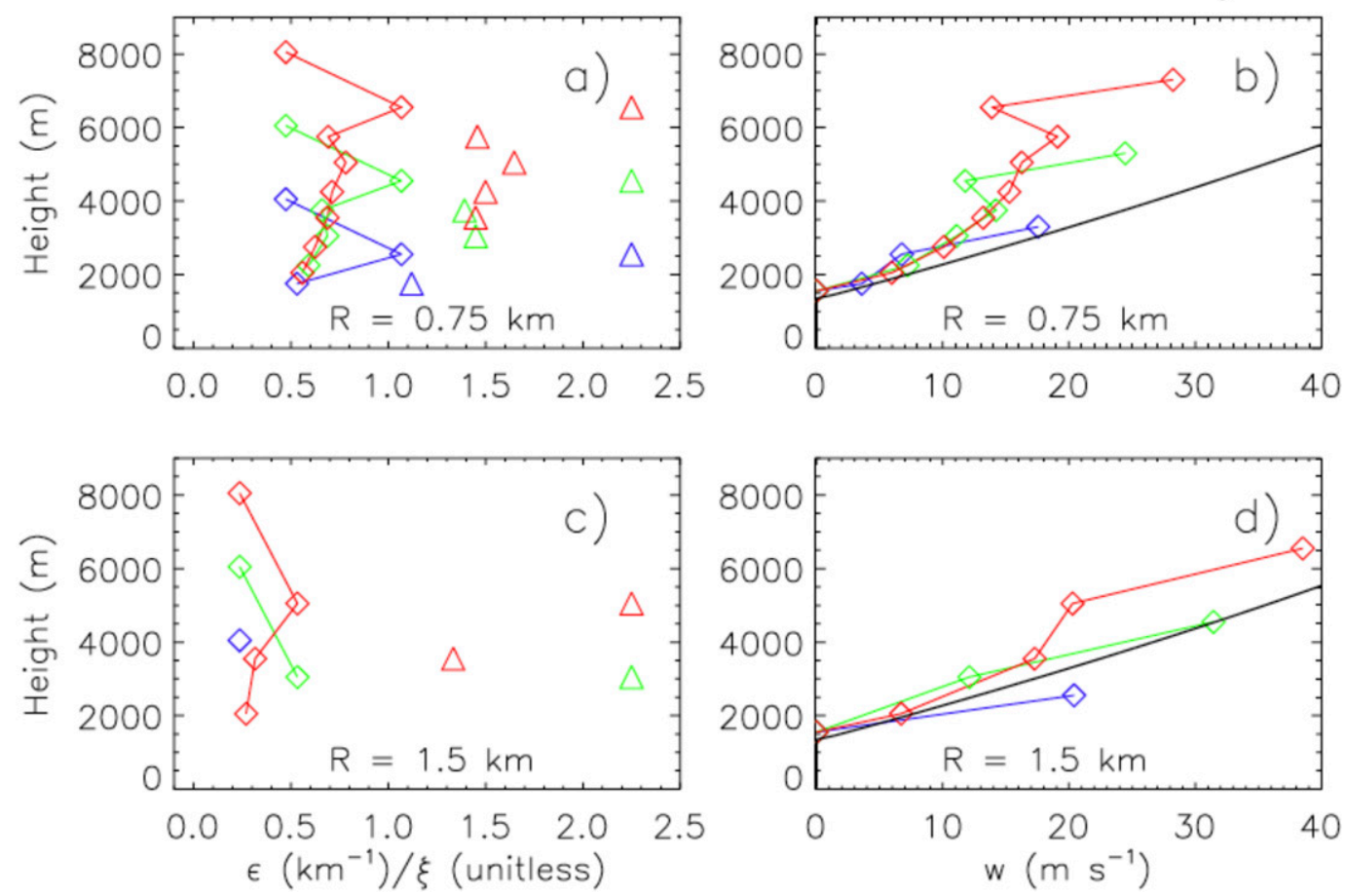

FIG. 12. As in Fig. 11, but for the moist environment with $R_{H}=0.85$.

The relationship between $\varepsilon$ and $B w^{-2}$ warrants further discussion. A positive relationship has been proposed based on the idea that the acceleration of entrained environmental air into the updraft requires conversion from buoyant to $w$ kinetic energy, whose ratio is proportional to $B w^{-2}$ (Gregory 2001; Del Genio and $\mathrm{Wu} 2010)$. The implication is that large entrainment rates require relatively large $B w^{-2}$, leading to an argument that $\psi \equiv \varepsilon /\left(B w^{-2}\right)$ should be approximately constant (Gregory 2001). However, mixing of updraft and environmental air also leads to dilution of $B$ itself, which complicates this idea. Hernandez-Deckers and Sherwood (2018) found that $B w^{-2}$ explained the second largest fraction of $\varepsilon$ variance after $R^{-1}$, about $15 \%$, in contrast to nearly zero correlation here. However, $\varepsilon$ and $B w^{-2}$ are positively correlated here when the data are segmented by height, with $r_{\text {corr }}$ between 0.434 and 0.570 for $4 \leq z \leq 7 \mathrm{~km}$ (Table 1 ). Note that correlations of $\varepsilon$ with the other convective parameters $\left(B, w, z^{-1}, R^{-1}, R_{H}\right)$ are similar whether all heights are considered or the data are segmented by height (not shown). The lack of correlation between $\varepsilon$ and $B w^{-2}$ considering all heights is explained by values of $\psi$ that are not constant, and in fact they increase sharply with height from 0.25 at $2 \mathrm{~km}$ to 2.65 at $7 \mathrm{~km}$ (rightmost column in Table 1). These $\psi$ values, which can be derived simply by taking the ratio of the theoretical expressions for $B$ and those for $w^{2}$, are remarkably consistent with profiles of $\psi$ from the LES of Del Genio and Wu (2010, see their Fig. 9).

From the theoretical expressions, strong positive correlation between $\varepsilon$ and $B w^{-2}$ when the data are segmented by height occurs definitively because of momentum entrainment. The dilution of $B$ in effect reduces both $B$ and $w^{2}$, while momentum mixing only directly dilutes $w^{2}$. Thus, for a given $\varepsilon, w^{2}$ is reduced relatively more than $B$ because of momentum mixing. This is demonstrated by recalculating correlation coefficients but neglecting momentum entrainment in the expressions for $w$ (Table 1). This test leads to little correlation below $6 \mathrm{~km}$ and weakly negative correlations at 6 and $7 \mathrm{~km}$. Finally, we note the parameter $\psi$ increases with height (Table 1) simply because $w$ depends on the integral of the various momentum forcings, including $B$, as air parcels rise. Thus, differences in the effects of mixing on $B$ and $w^{2}$ owing to momentum entrainment accumulate and increase $\psi$ over time/height.

Finally, we comment on the negative correlation between $\varepsilon$ and $w$ (or similarly, the positive correlation between $\varepsilon$ and $w^{-1}$, not shown, which has a correlation coefficient of 0.243 from the theoretical model). Similar relationships have also been found in previous modeling (e.g., Neggers et al. 2002; Lu et al. 2016; Zhang et al. 2016; Anber et al. 2019) and observational (Lu et al. 2016) studies, with generally larger correlations than found here. A complicating factor in interpreting such relationships is that while $w$ might be expected to influence $\varepsilon$, $\varepsilon$ also directly drives changes in $w$ through momentum mixing and especially buoyancy dilution. Indeed, in our simple theoretical model negative correlation between $\varepsilon$ and $w$ and positive correlation between $\varepsilon$ and $w^{-1}$ arise almost entirely from the direct effects of changes in dilution mediated by other parameters, particular $R$. Otherwise, $w$ itself has a limited impact 

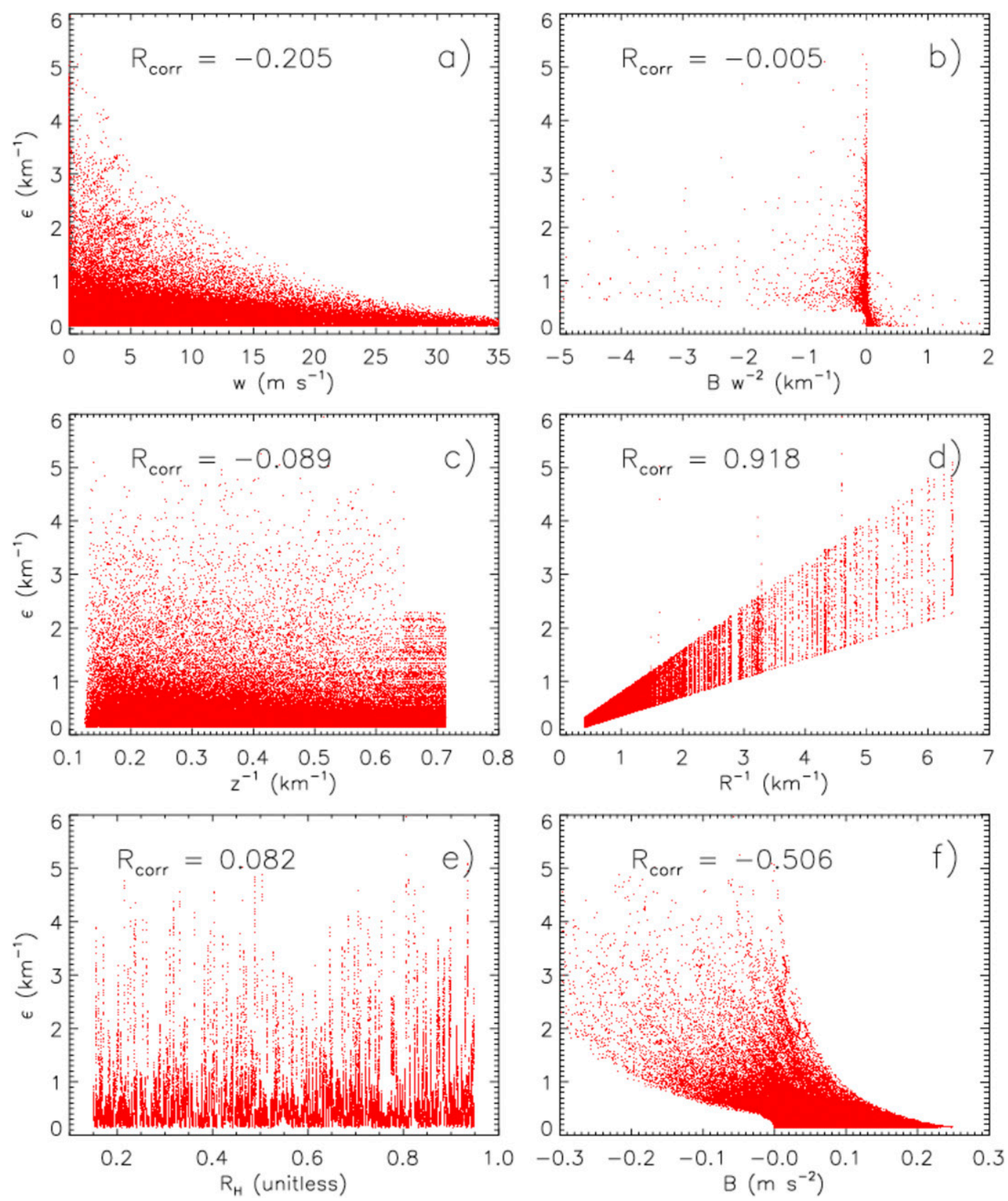

FIG. 13. Scatterplots of fractional entrainment rate $\varepsilon$ ( $y$ axis) with various quantities ( $x$ axis): (a) vertical velocity $w$, (b) the ratio of buoyancy and vertical velocity squared $B / w^{2}$, (c) inverse height $z^{-1}$, (d) inverse updraft radius $R^{-1}$, (e) environmental relative humidity $R_{H}$, and (f) buoyancy $B$. Each red dot indicates a data point obtained from the theoretical expressions by randomly sampling over ranges of $R, R_{H}$, updraft top height, and $z$ (see text for details). Linear correlation coefficients are shown in each plot.

in driving variability in $\varepsilon$ (this can be parsed in our model, which is one of the main benefits of a highly simplified framework). Larger $w$ decreases the time for mixing to occur for rising parcels in our model, which has been invoked to explain an inverse relationship between $\varepsilon$ and $w$ (e.g., Neggers et al. 2002; Anber et al. 2019); however, this effect is compensated by increased horizontal shear and larger mixing coefficients when $w$ is larger.

\section{Summary and conclusions}

In this paper, theoretical expressions for a passive scalar, buoyancy, and vertical velocity at different heights within growing moist updrafts were obtained. Overall, these expressions provide a simple quantitative model for cumulus convection, and they compare well to the numerical simulations in Part II. The theoretical expressions show how the thermal chain structure, with updrafts composed of rising thermals in 
TABLE 1. Values of linear correlation coefficient $r_{\text {corr }}$ for bulk fractional entrainment rate $\varepsilon$ and $B / w^{2}$ from the full theoretical expressions ("full") and neglecting momentum entrainment ("no-mom"). The last column shows mean values of nondimensional $\psi=\varepsilon w^{2} / B$ plus and minus one standard deviation from the full theoretical expressions. The row labeled "all" shows results for randomly sampled $R$, $R_{H}$, updraft-top height, and five randomly sampled heights between the LFC ( $\left.\sim 1400 \mathrm{~m}\right)$ and the updraft-top height (see text for details). The other rows show results for specific heights as indicated.

\begin{tabular}{cccc}
\hline \hline Height $(\mathrm{km})$ & "Full" $\varepsilon-\left(B / w^{2}\right) r_{\text {corr }}$ & "No-mom" $\varepsilon-\left(B / w^{2}\right) r_{\text {corr }}$ & $\psi$ \\
\hline All & -0.005 & -0.005 & $0.98 \pm 2.27$ \\
2 & -0.169 & -0.024 & $0.25 \pm 0.79$ \\
3 & 0.270 & -0.062 & $0.75 \pm 1.83$ \\
4 & 0.422 & -0.076 & $1.24 \pm 2.10$ \\
5 & 0.480 & -0.132 & $1.76 \pm 2.21$ \\
6 & 0.544 & -0.238 & $2.22 \pm 3.56$ \\
7 & 0.451 & -0.332 & $2.65 \pm 3.64$ \\
\hline
\end{tabular}

succession, arises from the governing equations of motion, mass continuity, and cloud thermodynamics. Thermal chains, as opposed to starting plumes (Turner 1962), are favored over a wide range of conditions. This is true particularly for a dry environment and moderate nondimensional updraft radius $R^{2} /(z L)$. When $R^{2} /(z L)$ is large and the environment is moist, starting plumes are favored. For small $R^{2} /(z L)$ and relatively dry conditions, the structure is akin to an isolated rising thermal. Fundamentally, the thermal chain structure emerges from the fact that $\partial w / \partial z$ is relatively large near the base of thermals, but is smaller below. This affects vertical profiles of dynamic entrainment and lateral turbulent mixing between environmental and cloudy updraft air. We showed how the thermal chain structure is predominately a feature of moist convection, owing to the close coupling between dynamic entrainment, locally enhanced mixing of $d r y$ environmental air, and reduction of buoyancy. The thermal chain structure with multiple organized $w$ maxima between the LFC and updraft top was much less evident when the entrained environmental air was humid $\left(R_{H}=100 \%\right)$.

Results suggest that thermal chains have a unique structure of fractional entrainment rate $\varepsilon$ compared to the traditional thermal and plume conceptual models. This occurs because of dynamic entrainment and enhanced turbulent mixing near the bottom of individual thermals in the chain, which results in up to a factor of $\sim 2$ local increase in $\varepsilon$, consistent with previous LES (Moser and Lasher-Trapp 2017) and evident in the simulations from Part II (though generally smaller in magnitude than in these simulations). The magnitude of $\varepsilon$ is similar between thermal chains in a dry environment and plume-like updrafts in a moist environment for a given $R$, although there are sharper maxima and minima below the primary thermal in thermals chains. Thus, large differences in how $B$ and $w$ evolve between thermal chains and plumes are mainly attributable to differences in the properties of entrained air, rather than $\varepsilon$.

Despite its simplicity, the theoretical model produces rather complicated behavior of $\varepsilon$. The strongest correlation is with $R^{-1}$, which is not surprising as it appears directly in the expressions for $\varepsilon$. There are also weak-to-moderate negative correlations of $\varepsilon$ with $B$ and $w$ owing to the fact that stronger entrainment directly dilutes and reduces $B$ and $w$. There is almost no correlation of $\varepsilon$ with $B w^{-2}$ considering all heights, but moderately positive correlations when partitioned by height consistent with previous LES (Del Genio and Wu 2010). The theoretical expressions provide a concise explanation for this relationship: increasing $\varepsilon$ leads to reduction of both $B$ and $w^{2}$ through buoyancy dilution, but $w^{2}$ decreases at a faster rate than $B$ because it is further reduced by momentum entrainment.

Overall, we have described a conceptual model for moist cumulus convection that goes beyond the traditional plume and thermal models. From the theoretical expressions, we showed conditions under which the various updraft structures are favored. These results also highlight the natural continuum of thermal, thermal chain, and starting plume moist convective structures. This work suggests that thermal chains are an intermediary regime between the thermal and starting plume modes, and occur over a wide range of updraft and environmental conditions. Most current convection parameterizations are based on the steady-state plume framework. The conceptual model here contrasts with this viewpoint and suggests nonsteady entrainment behavior associated with discrete rising thermals. The steps of building a moist convection scheme based directly on this model are nonobvious, but it could provide context for future parameterization development.

An important caveat of this work is that the effects of environmental vertical wind shear were neglected. It is well known that most deep, moist convection across the globe occurs in environments with nonnegligible vertical wind shear that often produces conditional instability through differential advection of temperature and moisture (Markowski and Richardson 2010). Vertical wind shear also encourages development of multicell, mesoscale systems that constitute much of the deep convection found across the globe (Nesbitt et al. 2006). We neglected environmental wind shear to derive tractable solutions. Recently, Peters et al. (2019) showed that asymmetry in the thermals' toroidal circulations caused by vertical wind shear induces a downward-directed dynamic pressure gradient force that increases their drag and slows ascent. Nonetheless, there were similar thermal chain-like updraft structures in both moderately sheared and unsheared environments. Additional study is needed to address this topic.

This framework also neglected the possible influence of environmental modification by earlier thermals that may 
influence the properties of air entrained by later thermals. This is similar to the idea of convective "preconditioning," whereby cumulus congestus detrain and moisten the atmosphere to promote later development of deep convection. The importance of this mechanism was suggested by some idealized modeling studies (Kuang and Bretherton 2006; Waite and Khouider 2010), but questioned by Hohenegger and Stevens (2013) based on observational analysis. It is difficult to generalize this effect in the theoretical model, and further work would be needed to include it.

In Part II we further explore the ideas presented herein. Specifically, we compare the theoretical expressions directly with results from axisymmetric numerical updraft simulations over a wide range of conditions. We also analyze thermal chainlike updraft structures in LES, and present results supporting the mechanisms proposed here explaining their occurrence.

Acknowledgments. This material is based on work supported by the National Center of Meteorology, Abu Dhabi, UAE, under the UAE Research Program for Rain Enhancement Science. This work was supported by the U.S. Department of Energy Atmospheric System Research (Grants DE-SC0016476, DE-SC0020104, and DE-SC0000246356). J. Peters's efforts were also partially supported by the National Science Foundation Grant AGS-1841674. A. Varble was supported by the U.S. Department of Energy (DOE) Office of Science Biological and Environmental Research as part of the Atmospheric System Research program. Pacific Northwest National Laboratory is operated by Battelle for the U.S. Department of Energy under Contract DE-AC05-76 RLO1830. W. Hannah's work was performed under the auspices of the U.S. DOE by Lawrence Livermore National Laboratory under Contract DE-AC52-07 NA27344. S. Giangrande's work was performed under the auspices of Brookhaven Science Associates, LLC, under Contract DE-SC0012704 with the U.S. DOE. The publisher by accepting the paper for publication acknowledges that the U.S. Government retains a nonexclusive, paid-up, irrevocable, worldwide license to publish or reproduce the published form of this paper, or allow others to do so, for U.S. Government purposes. We thank Dr. R. Rotunno for comments on an earlier version of the manuscript. The National Center for Atmospheric Research is sponsored by the National Science Foundation.

\section{REFERENCES}

Anber, U. M., S. E. Giangrande, L. J. Donner, and M. P. Jensen, 2019: Updraft constraints on entrainment: Insights from Amazonian deep convection. J. Atmos. Sci., 76, 2429-2442, https://doi.org/10.1175/JAS-D-18-0234.1, https://doi.org/10.1175/ JAS-D-18-0234.1.

Asai, T., and A. Kasahara, 1967: A theoretical study of compensating downward motions associated with cumulus clouds. J. Atmos. Sci., 24, 487-496, https://doi.org/10.1175/15200469(1967)024<0487:ATSOTC>2.0.CO;2.

Bechtold, P., M. Kohler, T. Jung, F. Doblas-Reyes, M. Leutbecher, M. J. Rodwell, F. Vitart, and G. Balsamo, 2008: Advances in simulating atmospheric variability with the ECMWF model: From synoptic to decadal time-scales. Quart. J. Roy. Meteor. Soc., 134, 1337-1351, https://doi.org/10.1002/QJ.289.
Betts, A. K., 1975: Parametric interpretation of trade-wind cumulus budget studies. J. Atmos. Sci., 32, 1934-1945, https://doi.org/ 10.1175/1520-0469(1975)032<1934:PIOTWC>2.0.CO;2.

Blyth, A. M., and J. Latham, 1993: Development of ice and precipitation in New Mexican summertime cumulus clouds. Quart. J. Roy. Meteor. Soc., 119, 91-120, https://doi.org/ 10.1002/qj.49711950905.

Böing, S. J., H. J. J. Jonker, W. A. Narawa, and A. P. Siebesma, 2014: On the deceiving aspects of mixing diagrams of deep cumulus convection. J. Atmos. Sci., 71, 56-68, https://doi.org/ 10.1175/JAS-D-13-0127.1.

Bretherton, C. S., J. R. McCaa, and H. Grenier, 2004: A new parameterization for shallow cumulus convection and its application to marine subtropical cloud-topped boundary layers. Part I: Description of 1D results. Mon. Wea. Rev., 132, 864-882, https://doi.org/10.1175/1520-0493(2004)132<0864: ANPFSC $>2.0 . \mathrm{CO} ; 2$.

Brown, M., and C. J. Nowotarski, 2019: The influence of lifting condensation level on low-level outflow and rotation in simulated supercell thunderstorms. J. Atmos. Sci., 76, 1349-1372, https://doi.org/10.1175/JAS-D-18-0216.1.

Bryan, G. H., and J. M. Fritsch, 2002: A benchmark simulation for moist nonhydrostatic numerical models. Mon. Wea. Rev., 130, 2917-2928, https://doi.org/10.1175/1520-0493(2002)130<2917: ABSFMN $>2.0 . \mathrm{CO} ; 2$.

- , and H. Morrison, 2012: Sensitivity of a simulated squall line to horizontal resolution and parameterization of microphysics. Mon. Wea. Rev., 140, 202-225, https://doi.org/10.1175/MWRD-11-00046.1.

—_, J. C. Wyngaard, and J. M. Fritsch, 2003: Resolution requirements for the simulation of deep moist updrafts. Mon. Wea. Rev., 131, 2394-2416, https://doi.org/10.1175/15200493(2003)131<2394:RRFTSO > 2.0.CO;2.

_, R. Rotunno, and J. M. Fritsch, 2007: Roll circulations in the convective region of a simulated squall line. J. Atmos. Sci., 64, 1249-1266, https://doi.org/10.1175/JAS3899.1.

Damiani, R., and G. Vali, 2007: Evidence for tilted toroidal circulations in cumulus. J. Atmos. Sci., 64, 2045-2060, https:// doi.org/10.1175/JAS3941.1.

$\longrightarrow,-$, and S. Haimov, 2006: The structure of thermals in cumulus from airborne dual-Doppler radar observations. J. Atmos. Sci., 63 , 1432-1450, https://doi.org/10.1175/JAS3701.1.

— , and Coauthors, 2008: The Cumulus, Photogrammetric, In Situ, and Doppler Observations experiment of 2006. Bull. Amer. Meteor. Soc., 89, 57-74, https://doi.org/10.1175/BAMS-89-1-57.

Dawe, J. T., and P. H. Austin, 2011a: The influence of the cloud shell on tracer budget measurements of LES cloud entrainment. J. Atmos. Sci., 68, 2909-2920, https://doi.org/10.1175/ 2011JAS3658.1.

$\longrightarrow$, and — 2011b: Interpolation of LES cloud surfaces for use in direct calculations of entrainment and detrainment. Mon. Wea. Rev., 139, 444-456, https://doi.org/10.1175/2010MWR3473.1.

Deardorff, J. W., 1972: Numerical investigation of neutral and unstable planetary boundary layers. J. Atmos. Sci., 29, 91-115, https://doi.org/10.1175/1520-0469(1972)029<0091:NIONAU> 2.0.CO;2.

Del Genio, A. D., and J. Wu, 2010: The role of entrainment in the diurnal cycle of continental convection. J. Climate, 23, 27222738, https://doi.org/10.1175/2009JCLI3340.1.

De Rooy, W. C., and A. P. Siebesma, 2010: Analytic expressions for entrainment and detrainment in cumulus convection. Quart. J. Roy. Meteor. Soc., 136, 1216-1227, https://doi.org/10.1002/ QJ.640. 
— - and Coauthors, 2013: Entrainment and detrainment in cumulus convection: An overview. Quart. J. Roy. Meteor. Soc., 139, 1-19, https://doi.org/10.1002/QJ.1959.

Emanuel, K. A., 1991: A scheme for representing cumulus convection in large-scale models. J. Atmos. Sci., 48, 2313-2329, https://doi.org/10.1175/1520-0469(1991)048<2313:ASFRCC > 2.0.CO;2.

- 1994: Atmospheric Convection. Oxford University Press, $588 \mathrm{pp}$.

Gregory, D., 2001: Estimation of entrainment rate in simple models of convective clouds. Quart. J. Roy. Meteor. Soc., 127, 53-72, https://doi.org/10.1002/QJ.49712757104.

Hannah, W. M., 2017: Entrainment versus dilution in tropical deep convection. J. Atmos. Sci., 74, 3725-3747, https://doi.org/ 10.1175/JAS-D-16-0169.1.

Hernandez-Deckers, D., and S. C. Sherwood, 2016: A numerical investigation of cumulus thermals. J. Atmos. Sci., 73, 4117-4136, https://doi.org/10.1175/JAS-D-15-0385.1.

— cumulus thermals. J. Atmos. Sci., 75, 3911-3924, https:// doi.org/10.1175/JAS-D-18-0077.1.

Heus, T., G. V. Dijk, H. J. J. Jonker, and H. E. A. V. den Akker, 2008: Mixing in shallow cumulus clouds studied by Lagrangian particle tracking. J. Atmos. Sci., 65, 2581-2597, https://doi.org/ 10.1175/2008JAS2572.1.

- , H. J. J. Jonker, H. E. A. van den Akker, E. J. Griffith, M. Koutek, and F. H. Post, 2009: A statistical approach to the life cycle analysis of cumulus clouds selected in a virtual reality environment. J. Geophys. Res., 114, D06208, https://doi.org/ 10.1029/2008JD010917.

Hohenegger, C., and B. Stevens, 2013: Preconditioning deep convection with cumulus congestus. J. Atmos. Sci., 70, 448-464, https://doi.org/10.1175/JAS-D-12-089.1.

Jakob, C., and A. P. Siebesma, 2003: A new subcloud model for mass-flux convection schemes: Influence on triggering, updraft properties, and model climate. Mon. Wea. Rev., 131, 2765-2778, https://doi.org/10.1175/1520-0493(2003) $131<2765$ :ANSMFM>2.0.CO;2.[

Jeevanjee, N., 2017: Vertical velocity in the gray zone. J. $A d v$. Model. Earth Syst., 9, 2304-2316, https://doi.org/10.1002/ 2017MS001059.

Kain, J. S., and J. M. Fritsch, 1990: A one-dimensional entraining/ detraining plume model and its application in convection parameterization. J. Atmos. Sci., 47, 2784-2802, https://doi.org/ 10.1175/1520-0469(1990)047<2784:AODEPM>2.0.CO;2.

Khairoutdinov, M., and D. Randall, 2006: High-resolution simulation of shallow-to-deep convection transition over land. J. Atmos. Sci., 63, 3421-3436, https://doi.org/10.1175/JAS3810.1.

Kirshbaum, D. J., 2011: Cloud-resolving simulations of deep convection over a heated mountain. J. Atmos. Sci., 68, 361-378, https://doi.org/10.1175/2010JAS3642.1.

— fields by mesoscale ascent. Quart. J. Roy. Meteor. Soc., 138, 2136-2150, https://doi.org/10.1002/qj.1954.

Koenig, L. R., 1963: The glaciating behavior of small cumulonimbus clouds. J. Atmos. Sci., 20, 29-47, https://doi.org/10.1175/15200469(1963)020<0029:TGBOSC $>2.0$. CO;2.

Kuang, Z., and C. S. Bretherton, 2006: A mass flux scheme view of a high-resolution simulation of a transition from shallow to deep cumulus convection. J. Atmos. Sci., 63, 1895-1909, https:// doi.org/10.1175/JAS3723.1.

Lebo, Z. J., and H. Morrison, 2015: Effects of horizontal and vertical grid spacing on mixing in simulated squall lines and implications for convective strength and structure. Mon. Wea. Rev., 143, 4355-4375, https://doi.org/10.1175/MWR-D15-0154.1.

Leger, J., J.-P. Lafore, J.-M. Piriou, and J.-F. Gueremy, 2019: A simple model of convective drafts accounting for the perturbation pressure term. J. Atmos. Sci., 76, 3129-3149, https:// doi.org/10.1175/JAS-D-18-0281.1.

Lu, C., Y. Liu, G. J. Zhang, X. Wu, S. Endo, L. Cao, Y. Li, and X. Guo, 2016: Improving parameterization of entrainment rate for shallow convection with aircraft measurements and large-eddy simulation. J. Atmos. Sci., 73, 761-773, https:// doi.org/10.1175/JAS-D-15-0050.1.

Markowski, P., and Y. Richardson, 2010: Mesoscale Meteorology in Midlatitudes. Wiley-Blackwell, 430 pp.

Morrison, H., 2016a: Impacts of updraft size and dimensionality on the perturbation pressure and vertical velocity in cumulus convection. Part I: Simple, generalized analytic solutions. J. Atmos. Sci., 73, 1441-1454, https://doi.org/10.1175/JAS-D-15-0040.1.

_ 2016b: Impacts of updraft size and dimensionality on the perturbation pressure and vertical velocity in cumulus convection. Part II: Comparison of theoretical and numerical solutions and fully dynamical simulations. J. Atmos. Sci., 73, 1455-1480, https://doi.org/10.1175/JAS-D-15-0041.1.

- 2017: An analytic description of the structure and evolution of growing deep cumulus updrafts. J. Atmos. Sci., 74, 809-834, https://doi.org/10.1175/JAS-D-16-0234.1.

— cent rate of moist convective thermals. J. Atmos. Sci., 75, 1699-1719, https://doi.org/10.1175/JAS-D-17-0295.1.

Morton, B. R., G. I. Taylor, and J. S. Turner, 1956: Turbulent gravitational convection from maintained and instantaneous sources. Proc. Roy. Soc. London, 234A, 1-23, https://doi.org/ 10.1098/rspa.1956.0011.

Moser, D. H., and S. Lasher-Trapp, 2017: The influence of successive thermals on entrainment and dilution in a simulated cumulus congestus. J. Atmos. Sci., 74, 375-392, https://doi.org/ 10.1175/JAS-D-16-0144.1.

Neggers, R. A. J., A. P. Siebesma, and H. J. J. Jonker, 2002: A multiparcel method for shallow cumulus convection. J. Atmos. Sci., 59, 1655-1668, https://doi.org/10.1175/1520-0469(2002) 059<1655:AMMFSC $>2.0$. CO 2 .

Nesbitt, S. W., R. Cifelli, and S. A. Rutledge, 2006: Storm morphology and rainfall characteristics of TRMM precipitation features. Mon. Wea. Rev., 134, 2702-2721, https://doi.org/ 10.1175/MWR3200.1.

Peters, J. M., 2016: The impact of effective buoyancy and dynamic pressure forcing on vertical velocities within two-dimensional updrafts. J. Atmos. Sci., 73, 4531-4551, https://doi.org/10.1175/ JAS-D-16-0016.1.

— W. M. Hannah, and H. Morrison, 2019: The influence of vertical wind shear on moist thermals. J. Atmos. Sci., 76, 16451659, https://doi.org/10.1175/JAS-D-18-0296.1.

, H. Morrison, W. M. Hannah, A. C. Varble, and S. E. Giangrande, 2020: Thermal chains in ascending moist updrafts: Part II: Analysis of idealized simulations. J. Atmos. Sci., 77, 3661-3681, https://doi.org/10.1175/JAS-D-19-0244.1.

Raymond, D. J., and A. M. Blyth, 1986: A stochastic mixing model for nonprecipitating cumulus clouds. J. Atmos. Sci., 43, 2708-2718, https://doi.org/10.1175/1520-0469(1986)043<2708:ASMMFN> 2.0.CO;2.

— , and - 1989: Precipitation development in a New Mexico thunderstorm. Quart. J. Roy. Meteor. Soc., 115, 1397-1423, https://doi.org/10.1002/qj.49711549011. 
Romps, D. M., 2010: A direct measure of entrainment. J. Atmos. Sci., 67, 1908-1927, https://doi.org/10.1175/2010JAS3371.1.

_- 2016: The stochastic parcel model: A deterministic parameterization of stochastically entraining convection. J. Adv. Model. Earth Syst., 8, 319-344, https://doi.org/ 10.1002/2015MS000537.

— , and A. B. Charn, 2015: Sticky thermals: Evidence for a dominant balance between buoyancy and drag in cloud updrafts. J. Atmos. Sci., 72, 2890-2901, https://doi.org/10.1175/ JAS-D-15-0042.1.

—_, and R. Oktem, 2015: Stereo photogrammetry reveals substantial drag on cloud thermals. Geophys. Res. Lett., 42, 5051-5057, https://doi.org/10.1002/2015GL064009.

Rousseau-Rizzi, R., D. J. Kirshbaum, and M. K. Yau, 2017: Initiation of deep convection over an idealized mesoscale convergence line. J. Atmos. Sci., 74, 835-853, https://doi.org/ 10.1175/JAS-D-16-0221.1.

Schlemmer, L., and C. Hohenegger, 2014: The formation of wider and deeper clouds as a result of cold-pool dynamics. J. Atmos. Sci., 71, 2842-2858, https://doi.org/10.1175/JAS-D-13-0170.1.

Scorer, R. S., 1957: Experiments on convection of isolated masses of buoyant fluid. J. Fluid Mech., 2, 583-594, https://doi.org/ 10.1017/S0022112057000397.

_ and F. H. Ludlam, 1953: Bubble theory of penetrative convection. Quart. J. Roy. Meteor. Soc., 79, 94-103, https://doi.org/ 10.1002/qj.49707933908.
Sherwood, S. C., D. Hernandez-Deckers, M. Colin, and F. Robinson, 2013: Slippery thermals and the cumulus entrainment paradox. J. Atmos. Sci., 70, 2426-2442, https://doi.org/10.1175/JAS-D-120220.1.

Simpson, J., and V. Wiggert, 1969: Models of precipitating cumulus towers. Mon. Wea. Rev., 97, 471-489, https://doi.org/10.1175/ 1520-0493(1969)097<0471:MOPCT>2.3.CO;2.

Turner, J. S., 1962: The 'starting plume' in neutral surroundings. J. Fluid Mech., 13, 356-368, https://doi.org/10.1017/ S0022112062000762.

Varble, A., and Coauthors, 2014: Evaluation of cloud-resolving and limited area model intercomparison simulations using TWPICE observations: 1. Deep convective updraft properties. J. Geophys. Res. Atmos., 119, 13 891-13 918, https://doi.org/ 10.1002/2013JD021371.

Waite, M. L., and B. Khouider, 2010: The deepening of tropical convection by congestus preconditioning. J. Atmos. Sci., 67, 2601-2615, https://doi.org/10.1175/2010JAS3357.1.

Weisman, M. L., and J. B. Klemp, 1982: The dependence of numerically simulated convective storms on vertical wind shear and buoyancy. Mon. Wea. Rev., 110, 504-520, https://doi.org/ 10.1175/1520-0493(1982)110<0504:TDONSC > 2.0.CO;2.

Zhang, G. J., X. Wu, X. Zeng, and T. Mitovski, 2016: Estimation of convective entrainment properties from a cloud-resolving model simulation during TWP-ICE. Climate Dyn., 47, 21772192, https://doi.org/10.1007/s00382-015-2957-7. 